\title{
Computational Cardiovascular Flow Analysis With the Variational Multiscale Methods
}

\author{
Kenji TAKIZAWA ${ }^{1, *}$, Yuri BAZILEVS ${ }^{2}$, Tayfun E. TEZDUYAR ${ }^{3,1}$, \\ Ming-Chen $H_{S U}{ }^{4}$ \\ ${ }^{1}$ Waseda University, Tokyo, Japan \\ ${ }^{2}$ Brown University, Providence, Rhode Island, USA \\ ${ }^{3}$ Rice University, Houston, Texas, USA \\ ${ }^{4}$ Iowa State University, Ames, Iowa, USA \\ *Corresponding Author: Kenji Takizawa (email: Kenji.Takizawa@tafsm.org) \\ (Received: 08-May-2019; accepted: 14-May-2019; published: 30-June-2019) \\ DOI: http://dx.doi.org/10.25073/jaec.201932.245
}

\begin{abstract}
Computational cardiovascular flow analysis can provide valuable information to medical doctors in a wide range of patientspecific cases, including cerebral aneurysms, aortas and heart valves. The computational challenges faced in this class of flow analyses also have a wide range. They include unsteady flows, complex cardiovascular geometries, moving boundaries and interfaces, such as the motion of the heart valve leaflets, contact between moving solid surfaces, such as the contact between the leaflets, and the fluid-structure interaction between the blood and the cardiovascular structure. Many of these challenges have been or are being addressed by the Space-Time Variational Multiscale (ST-VMS) method, Arbitrary Lagrangian-Eulerian VMS (ALE-VMS) method, and the VMS-based Immersogeometric Analysis (IMGA-VMS), which serve as the core computational methods, and the special methods used in combination with them. We provide an overview of the core and special methods and present examples of challenging computations carried out with these methods, including aorta and heart valve flow analyses.
\end{abstract}

\section{Keywords}

Cardiovascular flow analysis, Patientspecific blood flow analysis, Aorta, Heart valve, Space-Time VMS method, ALEVMS method, Immersogeometric Analysis

\section{Introduction}

Many agree that computational cardiovascular flow analysis can provide surgeons and medical doctors valuable information in a wide range of patient-specific cases, including cerebral aneurysms (see, e.g., [1]), treatment of cerebral aneurysms (see, e.g., [2]), aortas (see, e.g., [3]) and heart valves (see, e.g., $[4,5]$ ). The computational challenges faced in this class of flow analyses also have a wide range, many quite formidable. They include highly-unsteady flows and complex cardiovascular geometries. They also include moving boundaries and interfaces (MBI), such as the motion of the heart valve leaflets, contact between moving solid surfaces, such as the contact between the leaflets, and the fluid-structure interaction (FSI) between the blood and the cardiovascular structure. There is 
also a computational challenge associated with using medical-image-based arterial geometries. These geometries do not come from the zerostress state (ZSS) of the artery. A special method is needed to estimate the ZSS required in the FSI computation. The task becomes even more challenging for arteries with complex geometries, such as the aorta.

Many of these challenges have been or are being addressed by the Space-Time Variational Multiscale (ST-VMS) method [6], Arbitrary Lagrangian-Eulerian VMS (ALE-VMS) method [7], and the VMS-based Immersogeometric Analysis (IMGA-VMS) [4,8], which serve as the core computational methods, and the special methods used in combination with them. The special methods used in combination with the ST-VMS include the ST Slip Interface (STSI) method $[9,10]$, ST Topology Change (STTC) $[11,12]$ method, ST Isogeometric Analysis (ST-IGA) [6, 13, 14], integration of these methods, a general-purpose NURBS mesh generation method for complex geometries [15,16], and methods for estimating the ZSS of the artery [17-20]. The special methods used in combination with the ALE-VMS include weak enforcement of no-slip boundary conditions [21-23], "sliding interfaces" [24, 25] (the acronym "ST" will also indicate that) and backflow stabilization [26].

We will provide an overview of the core and special methods and present examples of challenging computations carried out with these methods, including aorta and heart valve flow analyses.

\section{Governing equations}

\subsection{Incompressible flow}

Let $\Omega_{t} \subset \mathbb{R}^{n_{\text {sd }}}$ be the spatial domain with boundary $\Gamma_{t}$ at time $t \in(0, T)$, where $n_{\mathrm{sd}}$ is the number of space dimensions. The subscript $t$ indicates the time-dependence of the domain.
The Navier-Stokes equations of incompressible flows are written on $\Omega_{t}$ and $\forall t \in(0, T)$ as

$$
\begin{array}{r}
\rho\left(\frac{\partial \mathbf{u}}{\partial t}+\mathbf{u} \cdot \nabla \mathbf{u}-\mathbf{f}\right)-\nabla \cdot \boldsymbol{\sigma}=\mathbf{0}, \\
\nabla \cdot \mathbf{u}=0,
\end{array}
$$

where $\rho, \mathbf{u}$ and $\mathbf{f}$ are the density, velocity and body force. The stress tensor $\boldsymbol{\sigma}(\mathbf{u}, p)=-p \mathbf{I}+$ $2 \mu \boldsymbol{\varepsilon}(\mathbf{u})$, where $p$ is the pressure, $\mathbf{I}$ is the identity tensor, $\mu=\rho \nu$ is the viscosity, $\nu$ is the kinematic viscosity, and the strain rate $\varepsilon(\mathbf{u})=$ $\left(\boldsymbol{\nabla u}+(\boldsymbol{\nabla u})^{T}\right) / 2$. The essential and natural boundary conditions for Eq. (1) are represented as $\mathbf{u}=\mathbf{g}$ on $\left(\Gamma_{t}\right)_{\mathrm{g}}$ and $\mathbf{n} \cdot \boldsymbol{\sigma}=\mathbf{h}$ on $\left(\Gamma_{t}\right)_{\mathbf{h}}$, where $\mathbf{n}$ is the unit normal vector and $\mathbf{g}$ and $\mathbf{h}$ are given functions. A divergence-free velocity field $\mathbf{u}_{0}(\mathbf{x})$ is specified as the initial condition.

\subsection{Structural mechanics}

In this article we will not provide any of our formulations requiring fluid and structure definitions simultaneously; we will instead give reference to earlier journal articles where the formulations were presented. Therefore, for notation simplicity, we will reuse many of the symbols used in the fluid mechanics equations to represent their counterparts in the structural mechanics equations. To begin with, $\Omega_{t} \subset \mathbb{R}^{n_{\text {sd }}}$ and $\Gamma_{t}$ will represent the structure domain and its boundary. The structural mechanics equations are then written, on $\Omega_{t}$ and $\forall t \in(0, T)$, as

$$
\rho\left(\frac{\mathrm{d}^{2} \mathbf{y}}{\mathrm{d} t^{2}}-\mathbf{f}\right)-\nabla \cdot \boldsymbol{\sigma}=\mathbf{0},
$$

where $\mathbf{y}$ and $\boldsymbol{\sigma}$ are the displacement and Cauchy stress tensor. The essential and natural boundary conditions for Eq. (3) are represented as $\mathbf{y}=$ $\mathbf{g}$ on $\left(\Gamma_{t}\right)_{\mathrm{g}}$ and $\mathbf{n} \cdot \boldsymbol{\sigma}=\mathbf{h}$ on $\left(\Gamma_{t}\right)_{\mathbf{h}}$. The Cauchy stress tensor can be obtained from

$$
\sigma=J^{-1} \mathbf{F S F}^{T},
$$

where $\mathbf{F}$ and $J$ are the deformation gradient tensor and its determinant, and $\mathbf{S}$ is the second Piola-Kirchhoff stress tensor. It is obtained from the strain-energy density function $\varphi$ as follows:

$$
\mathbf{S} \equiv \frac{\partial \varphi}{\partial \mathbf{E}}
$$


where $\mathbf{E}$ is the Green-Lagrange strain tensor:

$$
\mathbf{E}=\frac{1}{2}(\mathbf{C}-\mathbf{I}),
$$

and $\mathbf{C}$ is the Cauchy-Green deformation tensor:

$$
\mathbf{C} \equiv \mathbf{F}^{T} \cdot \mathbf{F} .
$$

From Eqs. (5) and (6),

$$
\mathbf{S}=2 \frac{\partial \varphi}{\partial \mathbf{C}}
$$

\subsection{Fluid-structure interface}

In an FSI problem, at the fluid-structure interface, we will have the velocity and stress compatibility conditions between the fluid and structure parts. The details on those conditions can be found in Section 5.1 of [1].

\section{ST-VMS and ST-SUPS}

The Deforming-Spatial-Domain/Stabilized ST (DSD/SST) method [27-29] was introduced for computation of flows with MBI, including FSI. In MBI computations the DSD/SST functions as a moving-mesh method. Moving the fluid mechanics mesh to follow an interface enables mesh-resolution control near the interface and, consequently, high-resolution boundary-layer representation near fluid-solid interfaces. The stabilization components of the original DSD/SST are the Streamline-Upwind/Petrov-Galerkin (SUPG) [30] and Pressure-Stabilizing/PetrovGalerkin (PSPG) [27] stabilizations, which are used widely. Because of the SUPG and PSPG components, the original DSD/SST is now called "ST-SUPS." The ST-VMS is the VMS version of the DSD/SST. The VMS components of the ST-VMS are from the residual-based VMS (RBVMS) method [31-34]. The ST-VMS has two more stabilization terms beyond those in the ST-SUPS, and the additional terms give the method better turbulence modeling features. The ST-SUPS and ST-VMS, because of the higher-order accuracy of the ST framework (see $[6,35])$, are desirable also in computations without MBI

The ST-SUPS and ST-VMS have been applied to many classes of FSI, MBI and fluid mechanics problems (see [36] for a comprehensive summary). The classes of problems include spacecraft parachute analysis for the landing-stage parachutes [1,37-40], coverseparation parachutes [41] and the drogue parachutes [42-44], wind-turbine aerodynamics for horizontal-axis wind-turbine rotors [1,45-47], full horizontal-axis wind-turbines [48-51] and vertical-axis wind-turbines $[9,52,53]$, flappingwing aerodynamics for an actual locust $[1,13,54$, 55], bioinspired MAVs [49, 50, 56, 57] and wingclapping $[11,58]$, blood flow analysis of cerebral aneurysms [49,59], stent-treated aneurysms $[2,59,60]$, aortas $[3,61-63]$ and heart valves $[5,11,12,50,63-65]$, spacecraft aerodynamics $[41,66]$, thermo-fluid analysis of ground vehicles and their tires [64,67], thermo-fluid analysis of disk brakes [10], flow-driven string dynamics in turbomachinery [68-70], flow analysis of turbocharger turbines [14-16,71], flow around tires with road contact and deformation [64,72-74], fluid films [75], ram-air parachutes [76], and compressible-flow spacecraft parachute aerodynamics [77,78].

The ST computational methods have a relatively long track record in arterial FSI analysis, starting with computations reported in [79-82]. These were among the earliest arterial FSI computations, and the core method was the STSUPS. Many ST computations were also reported in the last 15 years. In the first 8 years of that period the ST computations were for FSI of abdominal aorta [83], carotid artery [83] and cerebral aneurysms [84-90]. In the last 7 years, the ST computations focused on even more challenging aspects of cardiovascular fluid mechanics and FSI, including comparative studies of cerebral aneurysms [49,91], stent treatment of cerebral aneurysms $[2,59,60,92,93]$, heart valve flow computation $[5,11,12,50,63,65]$, aorta flow analysis [3,61-63], and coronary arterial dynamics [94].

For more on the ST-VMS and ST-SUPS, see [1]. In the flow analyses presented here, the ST framework provides higher-order accuracy in a 
general context. The VMS feature of the STVMS addresses the computational challenges associated with the multiscale nature of the unsteady flow. The moving-mesh feature of the ST framework enables high-resolution computation near the moving heart valve leaflets.

\section{ALE-VMS, RBVMS and ALE-SUPS}

The ALE-VMS method [1,7,45,95-99] is the VMS version of the ALE [100]. It succeeded the ST-SUPS [27] and ALE-SUPS [101] and preceded the ST-VMS. The VMS components are from the RBVMS [31-34]. The ALE-VMS originated from the RBVMS formulation of incompressible turbulent flows proposed in [33] for nonmoving meshes, and may be thought of as an extension of the RBVMS to moving meshes. As such, it was presented for the first time in [7] in the context of FSI. To increase their scope and accuracy, the ALE-VMS and RBVMS are often supplemented with special methods, such as those for weakly-enforced noslip boundary conditions [21-23], "sliding interfaces" [24, 25] and backflow stabilization [26]. The ALE-SUPS, RBVMS and ALE-VMS have been applied to many classes of FSI, MBI and fluid mechanics problems. The classes of problems include ram-air parachute FSI [101], windturbine aerodynamics and FSI $[45,51-53,102-$ 108], more specifically, vertical-axis wind turbines $[52,53,109,110]$, floating wind turbines [111], wind turbines in atmospheric boundary layers $[52,53,108,112]$, and fatigue damage in wind-turbine blades [113], patient-specific cardiovascular fluid mechanics and FSI [7,114-119], biomedical-device FSI [4, 8,120-123], ship hydrodynamics with free-surface flow and fluid-object interaction [124,125], hydrodynamics and FSI of a hydraulic arresting gear [126, 127], hydrodynamics of tidal-stream turbines with free-surface flow [128], passive-morphing FSI in turbomachinery [129], bioinspired FSI for marine propulsion [130,131], bridge aerodynamics and fluidobject interaction [132-134], Recent advances in stabilized and multiscale methods may be found for stratified incompressible flows in [135], for divergence-conforming discretizations of in- compressible flows in [136], and for compressible flows with emphasis on gas-turbine modeling in [137].

For more information on the ALE-VMS, RBVMS and ALE-SUPS, see [1]. In the flow analyses presented here, the VMS feature of the ALEVMS addresses the computational challenges associated with the multiscale nature of the unsteady flow. The moving-mesh feature of the ALE framework enables high-resolution computation near the moving wall of a thoracic aorta.

\section{IMGA-VMS}

The IMGA was introduced in [8] as a geometrically flexible technique for solving FSI problems involving large, complex structural deformation and change of fluid-domain topology (e.g., structural contact). The motivating application is the simulation of heart valve function over a complete cardiac cycle. The method directly analyzes a spline representation of a thin structure by immersing it into a non-body-fitted discretization of the background fluid domain, and focuses on accurately capturing the immersed design geometry within non-body-fitted analysis meshes. A new semi-implicit numerical method, which we now refer to as the Dynamic Augmented Lagrangian (DAL) approach [138], was introduced in [8] for weakly enforcing constraints in time-dependent immersogeometric FSI problems. A mixed ALE-VMS/IMGA-VMS (ALE-IMGA-VMS) method was developed in [4] in the framework of the Fluid-Solid InterfaceTracking/Interface-Capturing Technique [139]; a single computation combines a body-fitted, moving-mesh treatment of some fluid-structure interfaces, with a non-body-fitted treatment of others. This approach enables us to simulate the FSI of a bioprosthetic heart valve (BHV) in a deforming artery over the entire cardiac cycle under physiological conditions, and study the effect of arterial-wall elasticity on valve dynamics [4]. The DAL-based ALE-IMGA-VMS was integrated with Computer-Aided Design (CAD) for heart-valve analysis in [123] with a thorough comparison between dynamic and FSI computations. An anisotropic constitutive modeling of BHV leaflets for immersogeometric FSI, based 
on the Kirchhoff-Love shell formulation for general hyperelastic materials [140], is proposed in [141]. A divergence-conforming formulation of incompressible flow, which gives a pointwise divergence-free velocity field everywhere in the domain, is elegantly addressing the mass loss error across the valve interface in [142]. In addition, stable coupling strategies and suitable definition of Lagrange multipliers for the DAL numerical approach were proposed and analyzed in [143-145]. Finally, the FSI framework of ALE-IMGA-VMS was employed in patientspecific valve design in [146]. The DAL-based IMGA has also been combined with surrogate modeling in [127] for an efficient and effective use of FSI to optimize the design of a hydraulic arresting gear.

\section{ST-SI}

The ST-SI was introduced in [9], in the context of incompressible-flow equations, to retain the desirable moving-mesh features of the ST-VMS and ST-SUPS when we have spinning solid surfaces, such as a turbine rotor. The mesh covering the spinning surface spins with it, retaining the high-resolution representation of the boundary layers. The starting point in the development of the ST-SI was the version of the ALE-VMS for computations with sliding interfaces [24,25]. Interface terms similar to those in the ALE-VMS version are added to the ST-VMS to account for the compatibility conditions for the velocity and stress at the SI. That accurately connects the two sides of the solution. An ST-SI version where the SI is between fluid and solid domains was also presented in [9]. The SI in this case is a "fluid--solid SI" rather than a standard "fluid-fluid SI" and enables weak enforcement of the Dirichlet boundary conditions for the fluid. The ST-SI introduced in [10] for the coupled incompressible-flow and thermal-transport equations retains the high-resolution representation of the thermo-fluid boundary layers near spinning solid surfaces. These ST-SI methods have been applied to aerodynamic analysis of vertical-axis wind turbines [9,52,53], thermofluid analysis of disk brakes [10], flow-driven string dynamics in turbomachinery [68-70], flow analysis of turbocharger turbines [14-16, 71], flow around tires with road contact and deformation [64,72-74], fluid films [75], aerodynamic analysis of ram-air parachutes [76], and flow analysis of heart valves $[5,63,65]$.

In the ST-SI version presented in [9] the SI is between a thin porous structure and the fluid on its two sides. This enables dealing with the porosity in a fashion consistent with how the standard fluid-fluid SIs are dealt with and how the Dirichlet conditions are enforced weakly with fluid-solid SIs. This version also enables handling thin structures that have T-junctions. This method has been applied to incompressibleflow aerodynamic analysis of ram-air parachutes with fabric porosity [76]. The compressibleflow ST-SI methods were introduced in [77], including the version where the SI is between a thin porous structure and the fluid on its two sides. Compressible-flow porosity models were also introduced in [77]. These, together with the compressible-flow ST SUPG method [147], extended the ST computational analysis range to compressible-flow aerodynamics of parachutes with fabric and geometric porosities. That enabled ST computational flow analysis of the Orion spacecraft drogue parachute in the compressible-flow regime [77,78].

For more on the ST-SI, see $[9,10]$. In the computations here, the ST-SI is used in combination with the ST-TC, and we will describe the ST-SITC in Section 9. .

\section{Stabilization parameters}

The ST-SUPS, ALE-SUPS, RBVMS, ALEVMS, ST-VMS, ALE-SI and ST-SI all have some embedded stabilization parameters that play a significant role (see [1,9]). There are many ways of defining these stabilization parameters (for examples, see [9, 28, 29, 48,67,74, 148-164]). The stabilization-parameter definitions used in the computations reported in this article can be found from the references cited in the sections where those computations are described. 


\section{ST-TC}

The ST-TC [11,12] was introduced for movingmesh computation of flow problems with TC, such as contact between solid surfaces. Even before the ST-TC, the ST-SUPS and ST-VMS, when used with robust mesh update methods, have proven effective in flow computations where the solid surfaces are in near contact or create other near TC, if the nearness is sufficiently near for the purpose of solving the problem. Many classes of problems can be solved that way with sufficient accuracy. For examples of such computations, see the references mentioned in [11]. The ST-TC made moving-mesh computations possible even when there is an actual contact between solid surfaces or other TC. By collapsing elements as needed, without changing the connectivity of the "parent" mesh, the ST-TC can handle an actual TC while maintaining high-resolution boundary layer representation near solid surfaces. This enabled successful moving-mesh computation of heart valve flows [5,11,12,50,63-65], wing clapping [58], and flow around a rotating tire with road contact and prescribed deformation [64,72-74].

For more on the ST-TC, see $[11,12]$. In the computational analyses here, the ST-TC enables moving-mesh computation even with the TC created by the actual contact between the valve leaflets. It deals with the contact while maintaining high-resolution flow representation near the leaflet.

\section{ST-SI-TC}

The ST-SI-TC is the integration of the ST-SI and ST-TC. A fluid-fluid SI requires elements on both sides of the SI. When part of an SI needs to coincide with a solid surface, which happens for example when the solid surfaces on two sides of an SI come into contact or when an SI reaches a solid surface, the elements between the coinciding SI part and the solid surface need to collapse with the ST-TC mechanism. The collapse switches the SI from fluid-fluid SI to fluid-solid SI. With that, an SI can be a mixture of fluidfluid and fluid-solid SIs. With the ST-SI-TC, the elements collapse and are reborn independent of the nodes representing a solid surface. The ST-SI-TC enables high-resolution flow representation even when parts of the SI are coinciding with a solid surface. It also enables dealing with contact location change and contact sliding. This was applied to heart valve flow analysis $[5,63,65]$ and tire aerodynamics with road contact and deformation [72-74].

For more on the ST-SI-TC, see [5, 72]. In the computational analyses presented here, the STSI-TC enables high-resolution representation of the boundary layers even when the contact is between leaflets that are in mesh sectors connected by SIs. It enables contact location change and contact sliding between the leaflets.

\section{ST-IGA}

The ST-IGA is the integration of the ST framework with isogeometric discretization. It was introduced in [6]. Computations with the STVMS and ST-IGA were first reported in [6] in a $2 \mathrm{D}$ context, with IGA basis functions in space for flow past an airfoil, and in both space and time for the advection equation. Using higherorder basis functions in time enables getting full benefit out of using higher-order basis functions in space. This was demonstrated with the stability and accuracy analysis given in [6] for the advection equation.

The ST-IGA with IGA basis functions in time enables a more accurate representation of the motion of the solid surfaces and a mesh motion consistent with that. This was pointed out in $[6,35]$ and demonstrated in $[13,54,56]$. It also enables more efficient temporal representation of the motion and deformation of the volume meshes, and more efficient remeshing. These motivated the development of the ST/NURBS Mesh Update Method (STNMUM) [13,54,56]; the name was given in [48]. The STNMUM has a wide scope that includes spinning solid surfaces. With the spinning motion represented by quadratic NURBS in time, and with sufficient number of temporal patches for a full rotation, the circular paths are represented exactly. A "secondary mapping" $[1,6,13,35]$ en- 
ables also specifying a constant angular velocity for invariant speeds along the circular paths. The ST framework and NURBS in time also enable, with the "ST-C" method, extracting a continuous representation from the computed data and, in large-scale computations, efficient data compression $[10,64,67-70,165]$. The STNMUM and the ST-IGA with IGA basis functions in time have been used in many $3 \mathrm{D}$ computations. The classes of problems solved are flapping-wing aerodynamics for an actual locust $[1,13,54,55]$, bioinspired MAVs $[49,50,56,57]$ and wing-clapping $[11,58]$, separation aerodynamics of spacecraft [41], aerodynamics of horizontalaxis [48-51] and vertical-axis $[9,52,53]$ windturbines, thermo-fluid analysis of ground vehicles and their tires [64,67], thermo-fluid analysis of disk brakes [10], flow-driven string dynamics in turbomachinery [68-70], and flow analysis of turbocharger turbines [14-16,71].

The ST-IGA with IGA basis functions in space enables more accurate representation of the geometry and increased accuracy in the flow solution. It accomplishes that with fewer control points, and consequently with larger effective element sizes. That in turn enables using larger time-step sizes while keeping the Courant number at a desirable level for good accuracy. It has been used in ST computational flow analysis of turbocharger turbines [14-16,71], flow-driven string dynamics in turbomachinery [69,70], ramair parachutes [76], spacecraft parachutes [78], aortas [3,63], heart valves $[5,63,65]$, tires with road contact and deformation [73,74], and fluid films [75]. Using IGA basis functions in space is now a key part of some of the newest ZSS estimation methods [18-20,166] and related shell analysis [167].

For more on the ST-IGA, see $[1,14,54,76]$. In the computational flow analyses presented here, the ST-IGA enables more accurate representation of the cardiovascular geometries, increased accuracy in the flow solution, and using larger time-step sizes.

\section{ST-SI-TC-IGA}

The turbocharger turbine analysis [14-16, 71] and flow-driven string dynamics in turbomachinery $[69,70]$ were based on the integration of the ST-SI and ST-IGA. The IGA basis functions were used in the spatial discretization of the fluid mechanics equations and also in the temporal representation of the rotor and spinningmesh motion. That enabled accurate representation of the turbine geometry and rotor motion and increased accuracy in the flow solution. The IGA basis functions were used also in the spatial discretization of the string structural dynamics equations. That enabled increased accuracy in the structural dynamics solution, as well as smoothness in the string shape and fluid dynamics forces computed on the string.

The ram-air parachute analysis [76] and spacecraft parachute compressible-flow analysis [78] were based on the integration of the ST-IGA, the ST-SI version that weakly enforces the Dirichlet conditions, and the ST-SI version that accounts for the porosity of a thin structure. The STIGA with IGA basis functions in space enabled, with relatively few number of unknowns, accurate representation of the parafoil and parachute geometries and increased accuracy in the flow solution. The volume mesh needed to be generated both inside and outside the parafoil. Mesh generation inside was challenging near the trailing edge because of the narrowing space. The spacecraft parachute has a very complex geometry, including gores and gaps. Using IGA basis functions addressed those challenges and still kept the element density near the trailing edge of the parafoil and around the spacecraft parachute at a reasonable level.

The heart valve analysis $[5,63,65]$ was based on the integration of the ST-SI, ST-TC and ST-IGA. The ST-SI-TC-IGA, beyond enabling a more accurate representation of the geometry and increased accuracy in the flow solution, kept the element density in the narrow spaces near the contact areas at a reasonable level. When solid surfaces come into contact, the elements between the surface and the SI collapse. Before the elements collapse, the boundaries could be curved and rather complex, and the narrow 
spaces might have high-aspect-ratio elements. With NURBS elements, it was possible to deal with such adverse conditions rather effectively.

In computational analysis of flow around tires with road contact and deformation [74], the STSI-TC-IGA enables a more accurate representation of the geometry and motion of the tire surfaces, a mesh motion consistent with that, and increased accuracy in the flow solution. It also keeps the element density in the tire grooves and in the narrow spaces near the contact areas at a reasonable level. In addition, we benefit from the mesh generation flexibility provided by using SIs.

An SI provides mesh generation flexibility in a general context by accurately connecting the two sides of the solution computed over nonmatching meshes. This type of mesh generation flexibility is especially valuable in complex-geometry flow computations with isogeometric discretization, removing the matching requirement between the NURBS patches without loss of accuracy. This feature was used in the flow analysis of heart valves $[5,63,65]$, turbocharger turbines [14-16, 71], and spacecraft parachute compressible-flow analysis [78].

For more on the ST-SI-TC-IGA, see $[5,74]$. In the computations presented here, the ST-SI-TCIGA is used in the heart valve flow analysis, for the reasons given and as described in an earlier paragraph of this section.

\section{General-purpose NURBS mesh generation method}

To make the ST-IGA use, and in a wider context the IGA use, even more practical in computational flow analysis with complex geometries, NURBS volume mesh generation needs to be easier and more automated. To that end, a general-purpose NURBS mesh generation method was introduced in [15]. The method is based on multi-block-structured mesh generation with existing techniques, projection of that mesh to a NURBS mesh made of patches that correspond to the blocks, and recovery of the original model surfaces. The recovery of the original surfaces is to the extent they are suitable for accurate and robust fluid mechanics computations. The method is expected to retain the refinement distribution and element quality of the multi-block-structured mesh that we start with. Because there are ample good techniques and software for generating multi-blockstructured meshes, the method makes generalpurpose mesh generation relatively easy.

Mesh-quality performance studies for $2 \mathrm{D}$ and 3D meshes, including those for complex models, were presented in [16]. A test computation for a turbocharger turbine and exhaust manifold was also presented in [16], with a more detailed computation in [71]. The mesh generation method was used also in the pump-flow analysis part of the flow-driven string dynamics presented in [70] and in the aorta flow analysis presented in $[3,63]$. The performance studies, test computations and actual computations demonstrated that the general-purpose NURBS mesh generation method makes the IGA use in fluid mechanics computations even more practical.

For more on the general-purpose NURBS mesh generation method, see $[15,16]$. In the computations presented here, the method used in the aorta flow analysis.

\section{ZSS estimation methods}

The attempt to find a ZSS for the artery in the FSI computation was first made in a 2007 conference paper [168], where the concept of estimated zero-pressure (EZP) arterial geometry was introduced. The method introduced in [168] for calculating an EZP geometry was also included in a 2008 journal paper on ST arterial FSI methods [84], as "a rudimentary technique" for addressing the issue. It was pointed out in $[84,168]$ that quite often the medical-image-based geometries were used as arterial geometries corresponding to zero blood pressure, and that it would be more realistic to use the medical-image-based geometry as the arterial geometry corresponding to the time-averaged value of the blood pressure. Given the arterial geometry at the time- 
averaged pressure value, an estimated arterial geometry corresponding to zero blood pressure needed to be built. The special methods developed to address the issue include the newer EZP versions $[1,86,89,90,95]$ and the prestress technique introduced in [118], which was refined in [119] and presented also in [1,95].

A method for estimation of the element-based ZSS (EBZSS) was introduced in [17] in the context of finite element discretization of the arterial wall. The method has three main components. 1. An iteration technique, which starts with a calculated ZSS initial guess, is used for computing the EBZSS such that when a given pressure load is applied, the medicalimage-based target shape is matched. 2. A technique for straight-tube segments is used for computing the EBZSS so that we match the given diameter and longitudinal stretch in the target configuration and the "opening angle." 3 . An element-based mapping between the artery and straight-tube is extracted from the mapping between the artery and straight-tube segments. This provides the mapping from the arterial configuration to the straight-tube configuration, and from the estimated EBZSS of the straighttube configuration back to the arterial configuration, to be used as the ZSS initial guess for the iteration technique that matches the medicalimage-based target shape. Test computations with the method were also presented in [17] for straight-tube configurations with single and three layers, and for a curved-tube configuration with single layer. The method was used also in [94] in coronary arterial dynamics computations with medical-image-based time-dependent anatomical models.

The version of the EBZSS estimation method with isogeometric wall discretization, using NURBS basis functions, was introduced in [18]. With isogeometric discretization, we can obtain the element-based mapping directly, instead of extracting it from the mapping between the artery and straight-tube segments. Because all we need for the element-based mapping, including the curvatures, can be obtained within an element. With NURBS basis functions, we may be able to achieve a similar level of accuracy as with the linear basis functions, but using largersize and fewer elements, and the NURBS basis functions enable representation of more complex shapes within an element. The $2 \mathrm{D}$ test computations with straight-tube configurations presented in [18] showed how the EBZSS estimation method with NURBS discretization works. In [166], which is an expanded, journal version of [18], how the method can be used in a 3D computation where the target geometry is coming from medical image of a human aorta was also shown.

In the method introduced in [19], the estimate is based on T-spline discretization of the arterial wall and is in the form of integration-point-based ZSS (IPBZSS). The T-spline discretization enables dealing with complex arterial geometries, such as an aorta model with branches, while retaining the desirable features of isogeometric discretization. The IPBZSS is a convenient representation of the ZSS because with isogeometric discretization, especially with T-spline discretization, specifying conditions at integration points is more straightforward than imposing conditions on control points. The method has two main components. 1. An iteration technique, which starts with a calculated ZSS initial guess, is used for computing the IPBZSS such that when a given pressure load is applied, the medical-image-based target shape is matched. 2. A design procedure, which is based on the Kirchhoff-Love shell model of the artery, is used for calculating the ZSS initial guess.

In [20], the scope and robustness of the IPBZSS were increased method by introducing a new design procedure for the ZSS initial guess. The new design procedure has two features. a) An IPB shell-like coordinate system, which increases the scope of the design to general parametrization in the computational space. b) Analytical solution of the force equilibrium in the normal direction, based on the KirchhoffLove shell model, which places proper constraints on the design parameters. This increases the estimation accuracy, which in turn increases the robustness of the iterations and the convergence speed. To show how the new design procedure for the ZSS initial guess performs, the computations presented in [20] included 3D test computations with a straight tube and a Y-shaped tube. A 3D computation where the target geometry is coming from medical image 
of a human aorta was also presented in [20], and the aorta model included the branches.

For more on the ZSS estimation methods, see $[17,19,20,166]$. In the computations presented here, the newest method [20] is used in the ZSS estimate of the human aorta.

\section{ST computation: aortic-valve flow analysis}

This section is from [5].

\subsection{Geometry}

We have a typical aortic-valve model, such as the one in [58]. The model, shown in Figure 1, has three leaflets and one main outlet, corresponding to the beginning of the aorta. The leaflet motion is prescribed.
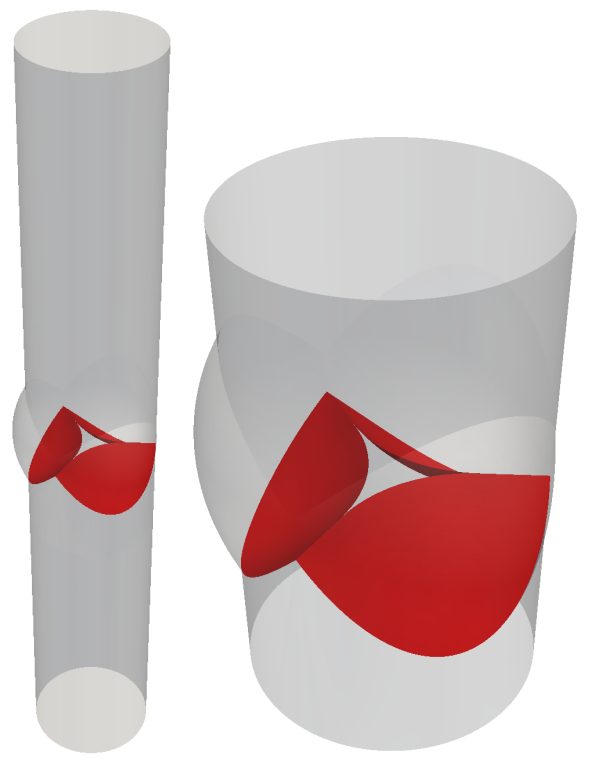

Fig. 1: Aortic-valve flow analysis. Model geometry. Aorta, leaflets, and sinuses. The left picture shows the entire computational domain, and the right picture is the zoomed view of the valve.

\subsection{Leaflet motion}

We consider two cases of leaflet motion: symmetric and asymmetric. In the symmetric case, the leaflet motions have 3 -fold rotational periodicity (i.e., 3-fold discrete rotational symmetry), and in the asymmetric case, they do not. We identify the individual leaflets as shown in Figure 2. The leaflet positions are defined by means of a pseudo-time parameter $\theta$, with the values 0 and 1 corresponding to the fully open and fully closed positions. The prescribed motion is given through $\theta$ as shown in Figure 3 for the symmetric case, and in Figure 4 for the asymmetric case.

\subsection{Mesh and flow conditions}

We create the mesh with five SIs, with three of them connecting the mesh sectors containing the leaflets in the valve region of the aorta (see Figure 5). The other two SIs, which are the top

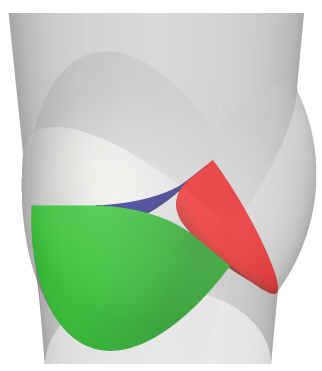

Fig. 2: Aortic-valve flow analysis. Leaflet identification. Leaflet 1 (red), 2 (green) and 3 (blue).

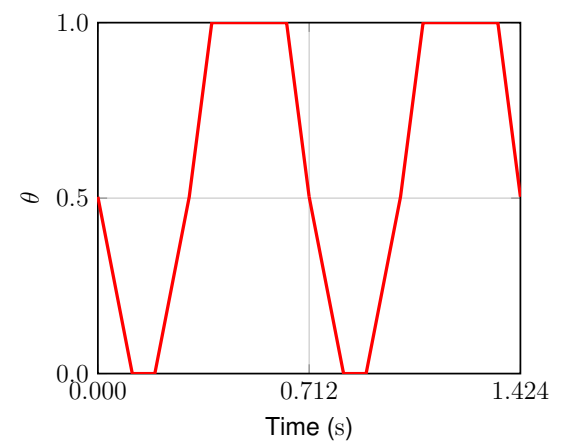

Fig. 3: Aortic-valve flow analysis. Symmetric leaflet motion. Pseudo-time parameter $\theta$ as a function of time for each of the three leaflets. 
and bottom circular planes in Figure 5, connect the meshes in the inlet and outlet regions to the valve region. They are for independent meshing in the inlet and outlet regions. The volume mesh is made of quadratic NURBS elements. The number of control points is 84,534 , and the number of elements is 54,000 . We prescribe the motion of the interior control points, and specify in each domain the master-slave mapping for all leaflet positions. Figure 6 shows a set of selected NURBS elements to illustrate how elements collapse. The boundary conditions are no-slip on the arterial walls and the leaflets, traction-free at the outflow boundary, and uniform velocity at the inflow boundary, with a temporal profile as shown in Figure 7. The cycle period is $0.712 \mathrm{~s}$. The no-slip condition on the arterial walls is enforced weakly.

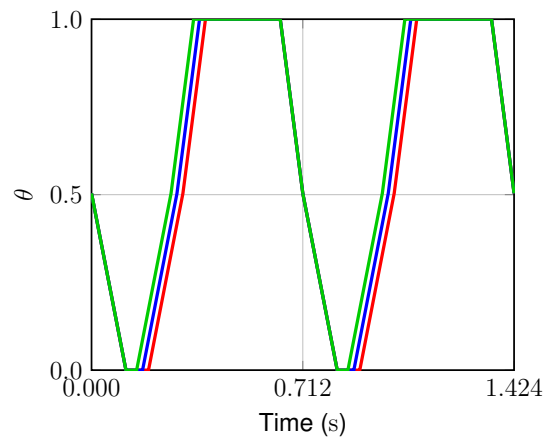

Fig. 4: Aortic-valve flow analysis. Asymmetric leaflet motion. Pseudo-time parameter $\theta$ as a function of time for each of the three leaflets.

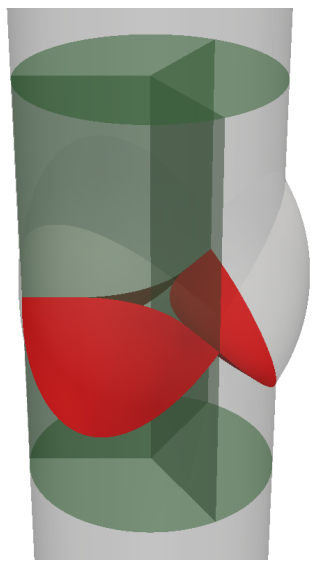

Fig. 5: Aortic-valve flow analysis. Aortic valve and the five SIs.

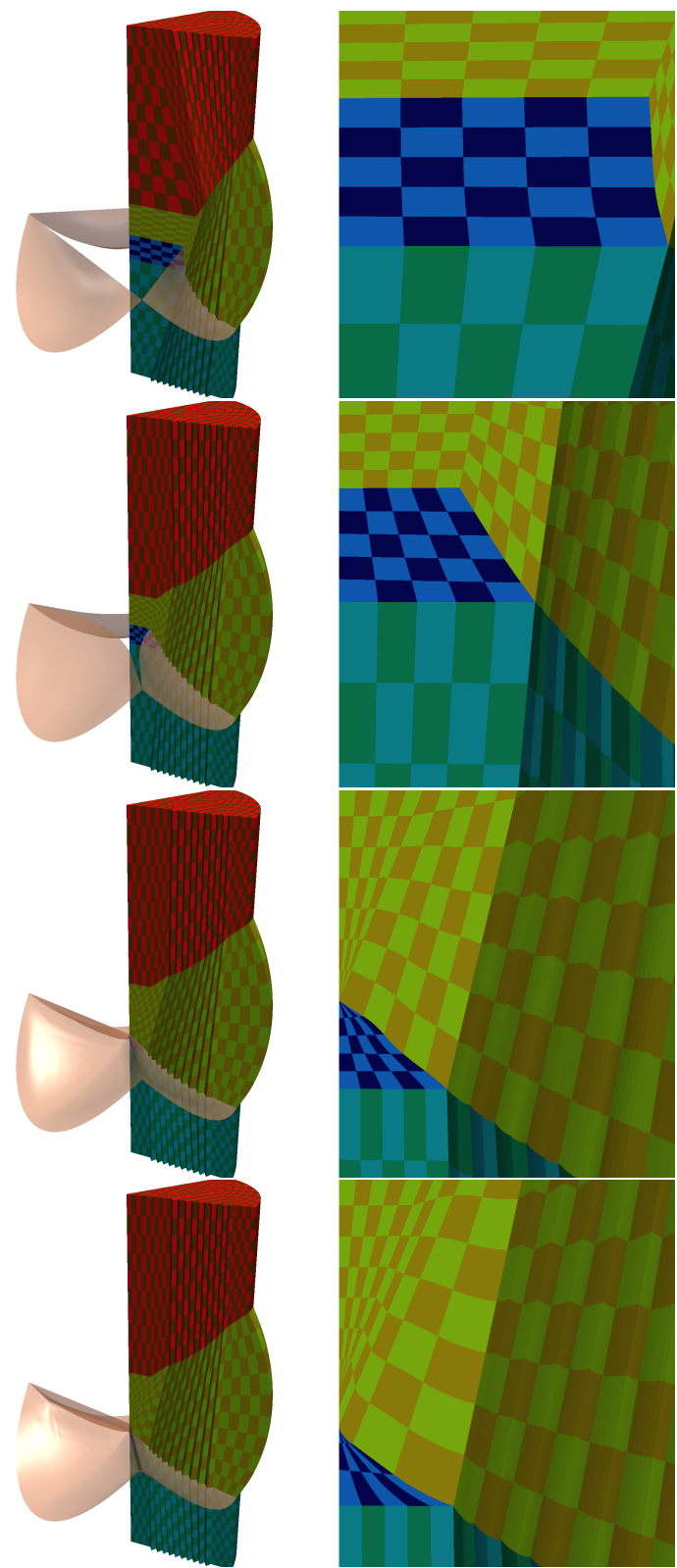

Fig. 6: Aortic-valve flow analysis. A set of selected NURBS elements, from when the valve is fully open $(t o p)$ to when it is fully closed (bottom). The corresponding $\theta$ values are $0.0,0.42,0.97$, and 1.0. The right pictures are the zoomed views around the leaflet.

\subsection{Computational conditions}

We use the ST-SUPS method. The time-step size is $4.00 \times 10^{-3} \mathrm{~s}$. There are three nonlinear iterations at each time step. The number 


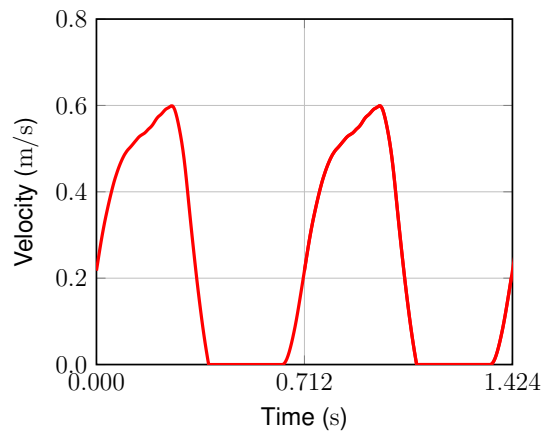

Fig. 7: Aortic-valve flow analysis. Inflow velocity (two cycles).

of GMRES iterations per nonlinear iteration is 300 .

\subsection{Results}

\section{1) Symmetric leaflet motion}

Figures 8 and 9 show the isosurfaces corresponding to a positive value of the second invariant of the velocity gradient tensor, colored by the velocity magnitude. The viewing angle is as we see the leaflets in Figure 2. The result shows how effectively the flow separation is captured near the leaflet edges and the solution is obtained as the surfaces come into contact.

Remark 1. We note that there is a thin vortex sheet near the aorta wall in the second and third row of pictures in Figure 9, which correspond to the fully closed positions of the valve. That is because full blocking of the flow requires a collapsed (i.e. zero-volume) element, which in turn requires that the control points of the element coalesce, and that is not happening for the elements with two edges on the leaflet edges and two edges on the aorta wall. In the current setting, the control points associated with the two edges on the aorta wall cannot coalesce.

We also report the the wall shear stress (WSS) on the leaflet surfaces. The viewing angle is as we see the leaflets in Figure 10. Figures 11 and 12 show the magnitude of the WSS on the upper and lower surfaces of the leaflets. As can be seen in the figures, the ST-SI-TC-IGA method gives a smooth WSS distribution, and

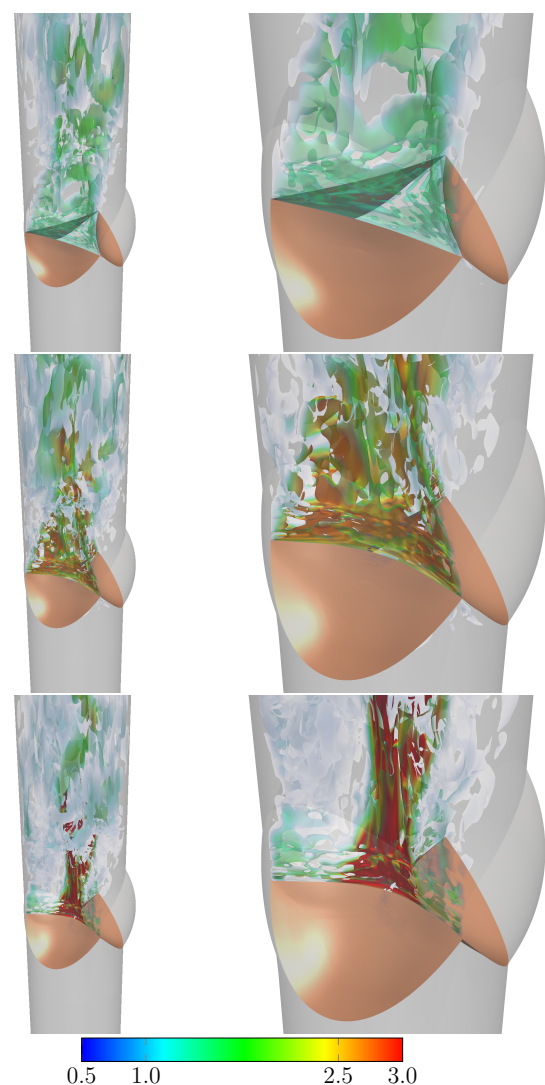

Fig. 8: Aortic-valve flow analysis. Symmetric leaflet motion. Isosurfaces corresponding to a positive value of the second invariant of the velocity gradient tensor, colored by the velocity magnitude $(\mathrm{m} / \mathrm{s})$. The frames are for $t=0.804,0.984$, and $1.028 \mathrm{~s}$.

the upper and lower WSS distributions are unrelated. The WSS is high on the lower surface near the edge, which is due to the flow jet.

\section{2) Asymmetric leaflet motion}

Figures 13 and 14 show the isosurfaces corresponding to a positive value of the second invariant of the velocity gradient tensor, colored by the velocity magnitude. The viewing angle is as we see the leaflets in Figure 2. In this case we have a biased flow jet due to the asymmetric leaflet closing. This can be seen from the third pair of pictures in Figure 13, and the first and second pair of pictures in Figure 14.

Figures 15 and 16 show the magnitude of the WSS on the upper and lower surfaces of the 


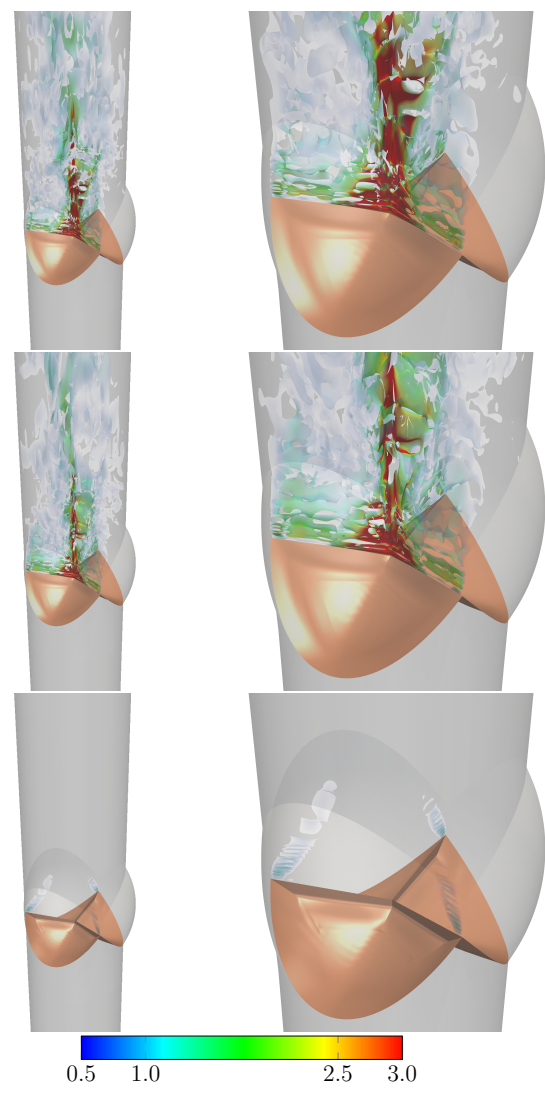

Fig. 9: Aortic-valve flow analysis. Symmetric leaflet motion. Isosurfaces corresponding to a positive value of the second invariant of the velocity gradient tensor, colored by the velocity magnitude $(\mathrm{m} / \mathrm{s})$. The frames are for $t=1.072,1.080$, and $1.252 \mathrm{~s}$.

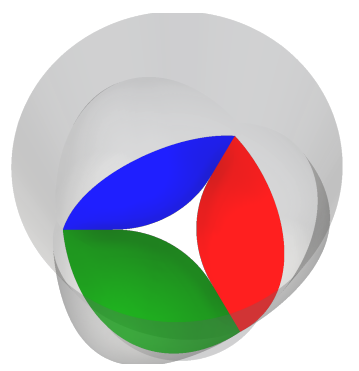

Fig. 10: Aortic-valve flow analysis. Viewing angle for reporting the WSS. The leaflet identification is same as in Figure 2.

leaflets. The viewing angle is as we see the leaflets in Figure 10.

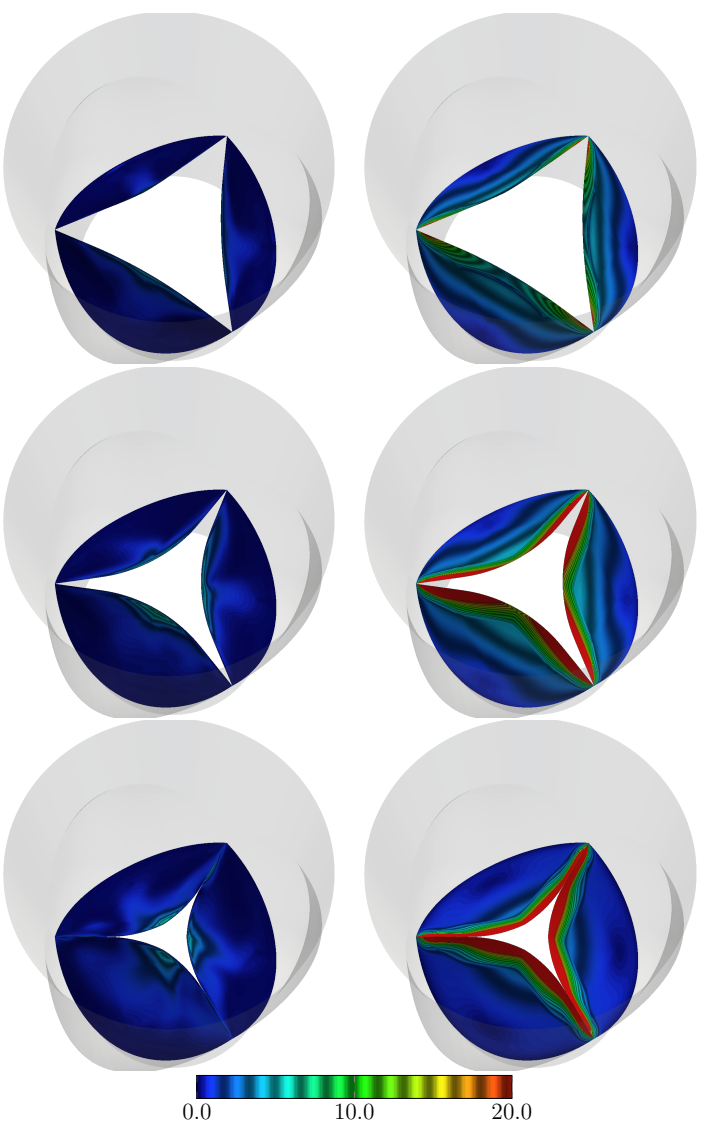

Fig. 11: Aortic-valve flow analysis. Symmetric leaflet motion. Magnitude of the WSS (Pa). Upper surface (left) and lower surface (right). The frames are for $t=0.804,0.984$, and $1.028 \mathrm{~s}$.

\section{ST computation: patient-specific aorta flow analysis}

This section is from [3].

We start with a geometry obtained from medical images and then use cubic T-splines to represent the surface. The density and kinematic viscosity of the blood are $1,050 \mathrm{~kg} / \mathrm{m}^{3}$ and $4.2 \times 10^{-6} \mathrm{~m}^{2} / \mathrm{s}$.

\subsection{Conditions}

The computational domain and boundary conditions are shown in Figure 17. The diameters are given in Table 1. The inflow flow rate, plug flow, 
Tab. 1: Patient-specific aorta flow analysis. Diameter $(\mathrm{mm})$ of the inlet and outlets. The outlets are listed in the order of closeness to the inlet.

\begin{tabular}{l|r|r|r|r|r|r}
\hline \hline & Inlet & Outlet 1 & Outlet 2 & Outlet 3 & Outlet 4 & Outlet 5 \\
\hline Diameter & 25.6 & 5.81 & 3.90 & 4.41 & 6.43 & 19.9 \\
\hline
\end{tabular}

is in Figure 18. The peak value of the average inflow velocity is $0.709 \mathrm{~m} / \mathrm{s}$. We estimate the outflows as distributed by Murray's law [169]:

$$
Q_{o} \propto D_{o}^{3}
$$

where $Q_{o}$ is the volumetric outflow rate, and the outlet diameter $D_{o}$ is defined based on the outlet area $A_{o}$ :

$$
D_{o}=2 \sqrt{\frac{A_{o}}{\pi}}
$$

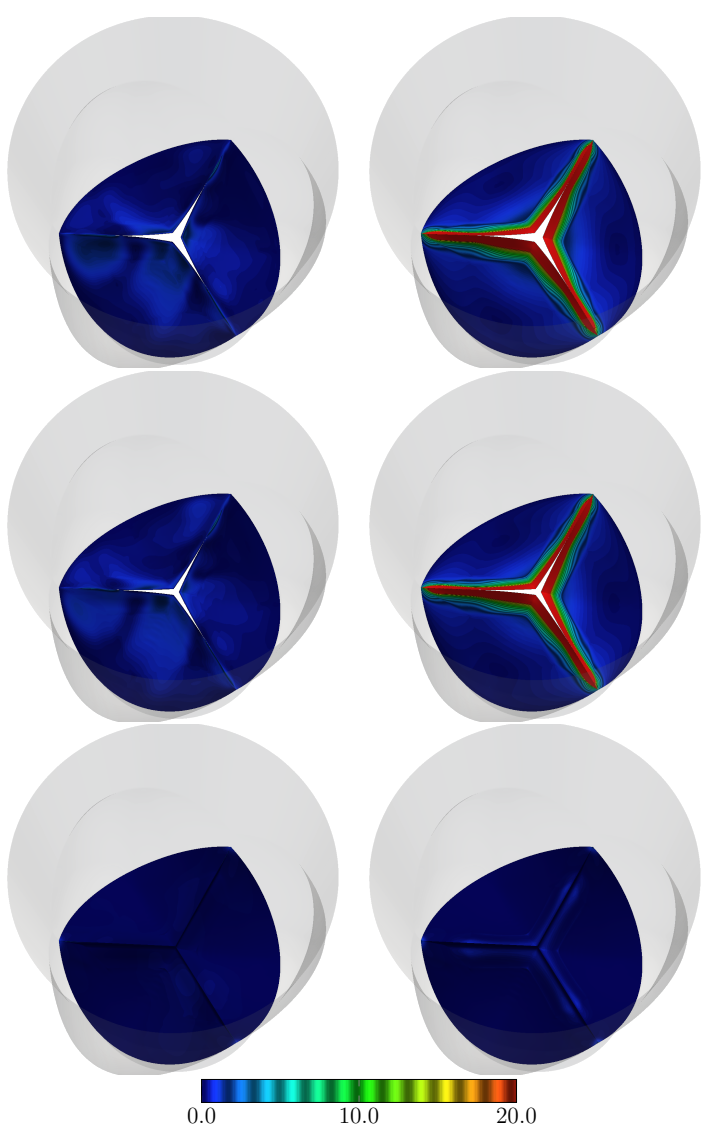

Fig. 12: Aortic-valve flow analysis. Symmetric leaflet motion. Magnitude of the WSS (Pa). Upper surface (left) and lower surface (right). The frames are for $t=1.072,1.080$, and $1.252 \mathrm{~s}$.
We form a plug flow profile at the smaller outlets, and the main outlet is set to traction free.

\subsection{Mesh}

We create a quadratic NURBS mesh from the T-spline surface, using the technique introduced in $[15,16]$. Figure 19 shows one of the NURBS patches and five of the patches together to illustrate the block-structured nature of the NURBS

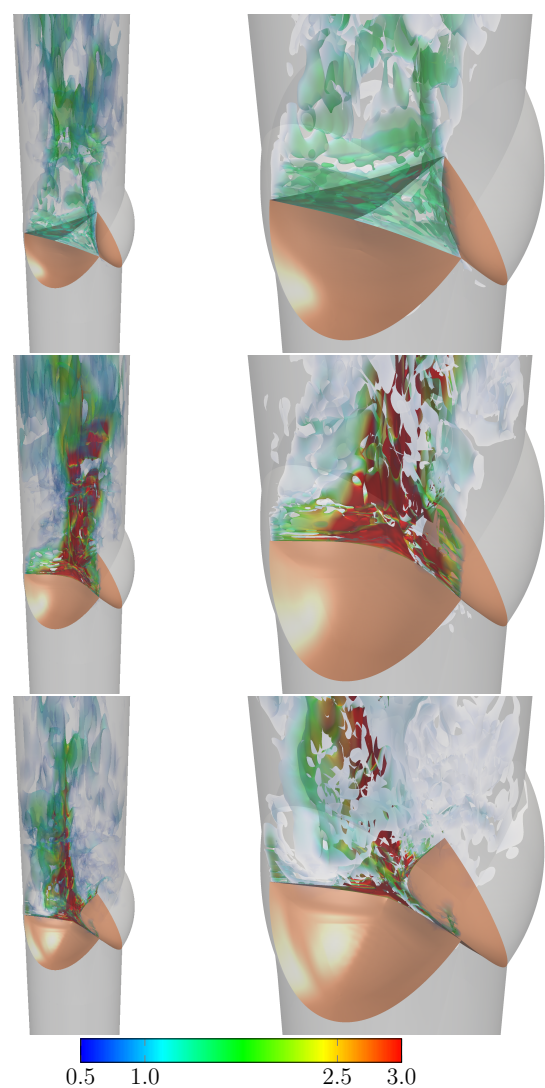

Fig. 13: Aortic-valve flow analysis. Asymmetric leaflet motion. Isosurfaces corresponding to a positive value of the second invariant of the velocity gradient tensor, colored by the velocity magnitude $(\mathrm{m} / \mathrm{s})$. The frames are for $t=0.804$, 0.984 , and $1.028 \mathrm{~s}$. 
mesh. The function space has only $C^{0}$ continuity between the patches. Figure 20 shows the base mesh. Figure 21 shows the base and refined meshes at the inlet. The meshes are refined by knot insertion, therefore the geometry is unchanged, and the basis functions for the coarser meshes are subsets of the basis functions for the finer meshes. The refinement is in the normal direction, and at each refinement, the element thickness is halved in half of the most refined layers. For the base mesh, the element thickness in the normal direction is approximately 1 $\%$ of the local diameter. There is no refinement in the tangential directions. During the refinement, the original plug flow profiles of the base mesh are retained. Table 2 shows the number of elements and control points.

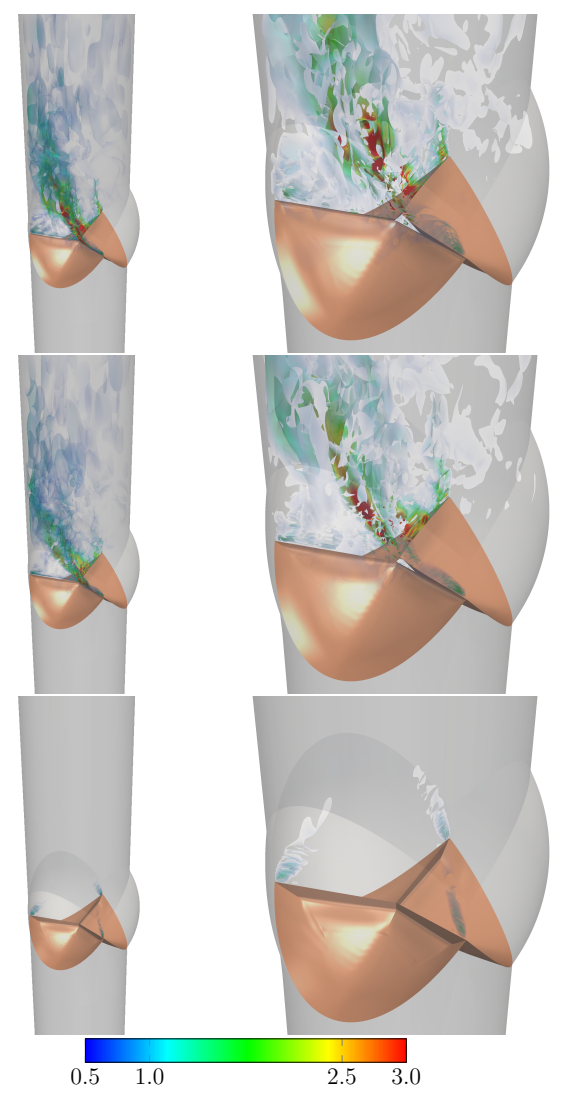

Fig. 14: Aortic-valve flow analysis. Asymmetric leaflet motion. Isosurfaces corresponding to a positive value of the second invariant of the velocity gradient tensor, colored by the velocity magnitude $(\mathrm{m} / \mathrm{s})$. The frames are for $t=1.072$, 1.080 , and $1.252 \mathrm{~s}$.
Tab. 2: Patient-specific aorta flow analysis. Number of control points $(n c)$ and element $(n e)$ for the quadratic NURBS meshes used in the computations.

\begin{tabular}{lcc} 
& $n c$ & $n e$ \\
\hline \hline Base Mesh & 202,497 & 151,513 \\
\hline Refinement Mesh 1 & 266,437 & 205,733 \\
\hline Refinement Mesh 2 & 330,377 & 259,953 \\
\hline Refinement Mesh 3 & 394,317 & 314,173 \\
\hline Refinement Mesh 4 & 458,257 & 368,393 \\
\hline
\end{tabular}

\subsection{Mesh refinement study}

We compute with the 5 meshes in Table 2 . The time-step sizes are $\Delta t=0.0025 \mathrm{~s}$ for Base Mesh and Refinement Mesh 1 and 2, and $\Delta t=$ $0.00125 \mathrm{~s}$ for Refinement Mesh 3 and 4 . The

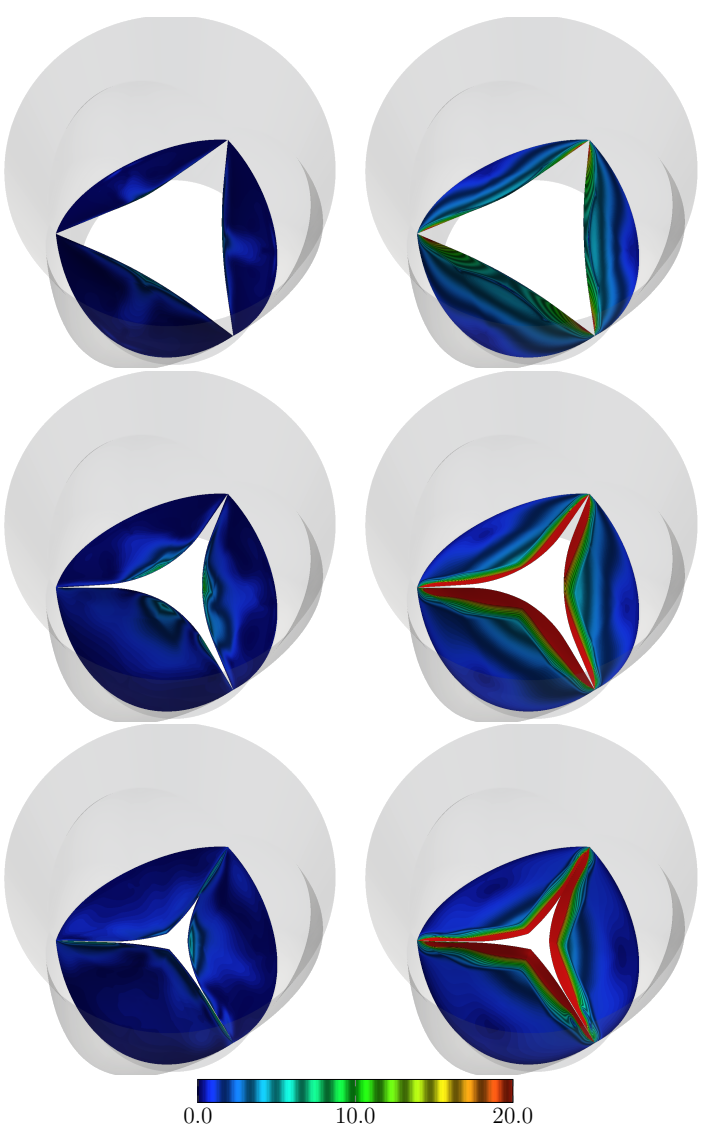

Fig. 15: Aortic-valve flow analysis. Asymmetric leaflet motion. Magnitude of the WSS $(\mathrm{Pa})$. Upper surface (left) and lower surface (right). The frames are for $t=0.804,0.984$, and $1.028 \mathrm{~s}$. 
number of nonlinear iterations per time step is 3 , and the number of GMRES iterations per nonlinear iteration is 800 for Base Mesh and Refinement Mesh 1, and 1,200, 1,400, and 1,600 for Refinement Mesh 2, 3, and 4, respectively. The ST-SUPS method is used and the stabilization parameters are those given by Eqs. (2.4)-(2.6), (2.8) and (2.10) in [9].

We first compute 9 cycles with Base Mesh, and the initial condition for the refined meshes is obtained by knot insertion. The solution reported here is for the 10th cycle. Figure 22 shows the solution computed with Refinement Mesh 4. At the peak flow rate a complex flow pattern is formed, and the vortex structure breaks down into smaller structures during the deceleration. The magnitude of the WSS $\left(\mathbf{h}_{\mathrm{v}}\right)$ at the peak flow rate is shown for each

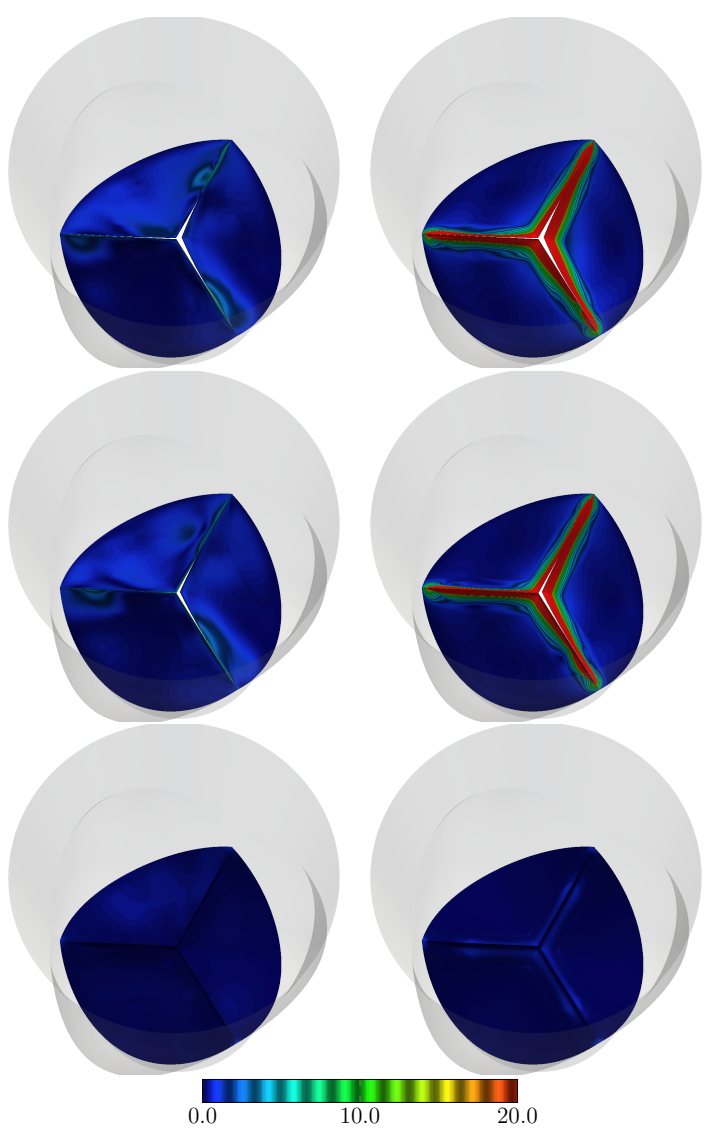

Fig. 16: Aortic-valve flow analysis. Asymmetric leaflet motion. Magnitude of the WSS (Pa). Upper surface (left) and lower surface (right). The frames are for $t=1.072,1.080$, and $1.252 \mathrm{~s}$. mesh in Figure 23. Qualitatively, all results are in good agreement, and the convergence can be seen with refinement. To quantify the mesh refinement level, we calculate the $y^{+}$value for the first-element thickness $h$ as

$$
y^{+}=\frac{u^{*} h}{\nu}
$$

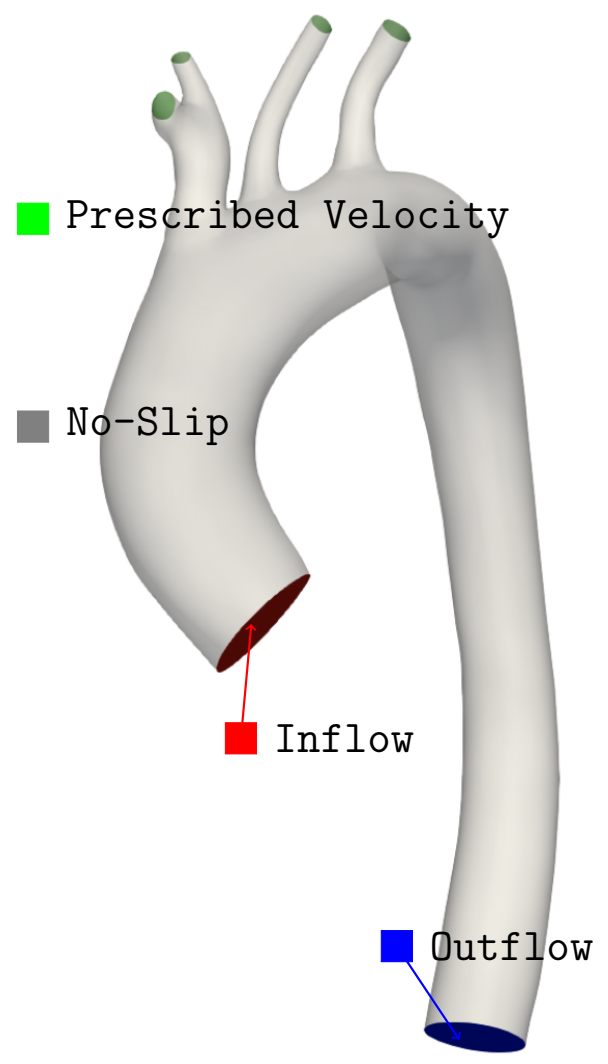

Fig. 17: Patient-specific aorta flow analysis. Geometry and boundary conditions.

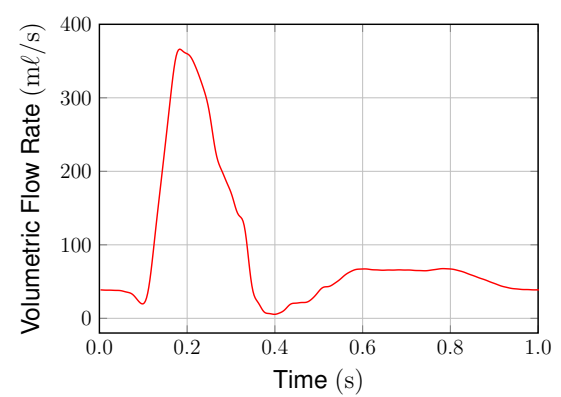

Fig. 18: Patient-specific aorta flow analysis. Volumetric flow rate at the inlet. 
where the friction velocity $u^{*}$ is based on the computed value of the WSS as follows:

$$
u^{*}=\sqrt{\frac{\left\|\mathbf{h}_{\mathrm{v}}^{h}\right\|}{\rho}} .
$$

Figure 24 shows the spatial distribution of $y^{+}$at the peak flow rate. It shows that for the meshes used here, $y^{+}$range is from approximate maximum 10 to less than 1. Comparing Figures 23 and 24 , we see that the WSS values computed over different meshes are in agreement where $y^{+} \leq 1$.

The time-averaged WSS magnitude (TAWSS) is shown in Figure 25, and Figure 26 shows the spatially-averaged WSS magnitude in a cycle.

Figure 27 shows the oscillatory shear index (OSI), defined as

$$
\mathrm{OSI}=\frac{1}{2}\left(1-\frac{\left\|\int_{0}^{T} \mathbf{h}_{\mathrm{v}}^{h} \mathrm{~d} t\right\|}{\int_{0}^{T}\left\|\mathbf{h}_{\mathrm{v}}^{h}\right\| \mathrm{d} t}\right) .
$$
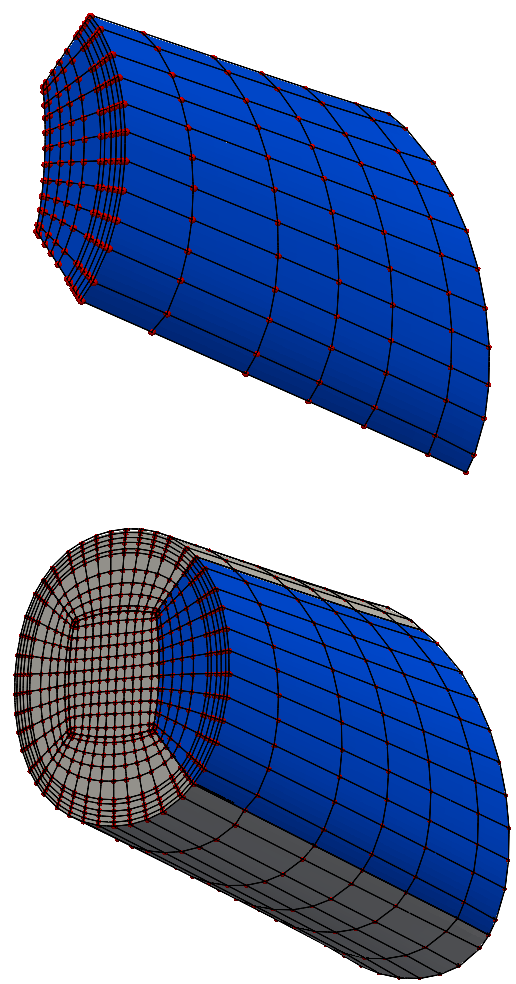

Fig. 19: Patient-specific aorta flow analysis. NURBS control mesh. One of the patches $(t o p)$ and five of the patches together (bottom).
Overall for OSI, even Base Mesh is in a good agreement with others. However, if we compare details such as branches, we see some difference even where $y^{+}$value is small. To see the flow differences, using the solution from Refinement Mesh 4 as the reference solution, we inspect the velocity difference $\left\|\mathbf{u}_{k}^{h}-\mathbf{u}_{4}^{h}\right\|$, where the subscripts indicate Base Mesh and Refinement Mesh $k$.

Remark 2. To calculate the velocity difference, all meshes and corresponding solutions are refined by using the knot-insertion technique, and

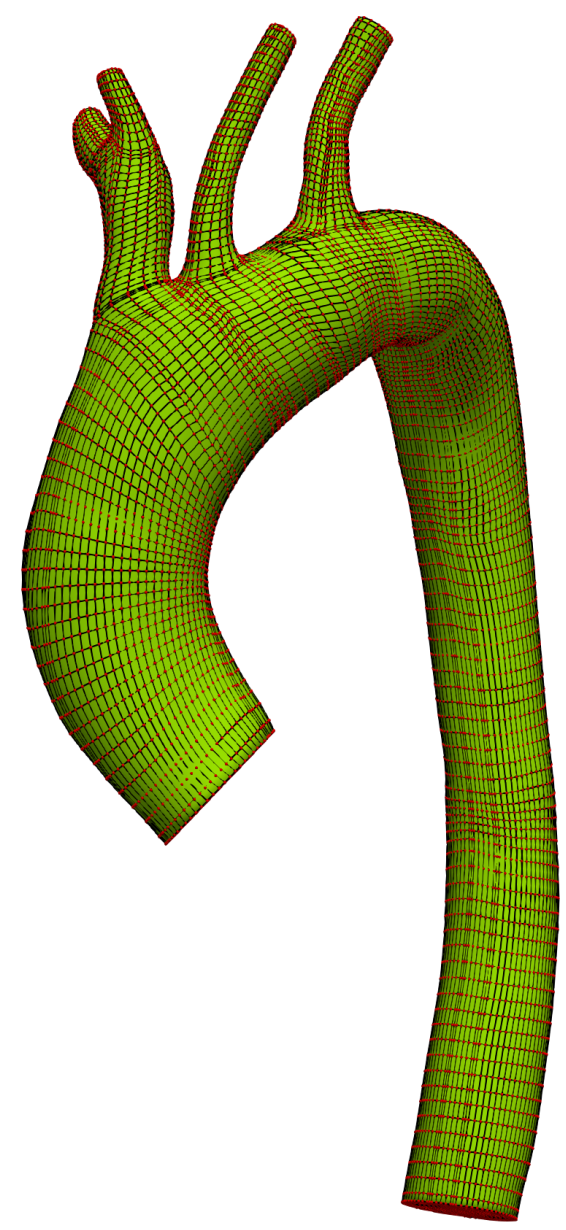

Fig. 20: Patient-specific aorta flow analysis. Base Mesh. Control mesh and surface (green). Red points are control points. 
the control variables are obtained based on Refinement Mesh 4. The visualization is done after taking the difference between the control variables, interpolating the vector, and taking its magnitude.

The spatial average of the difference is maximum at around $0.5 \mathrm{~s}$. This indicates that the vortex breakdown, due to the small-scale flow behavior that needs to be dealt with, would not be easy to resolve. Figure 28 shows the velocity difference at $0.5 \mathrm{~s}$.

In summary, good accuracy in the WSS magnitude can be obtained with locally good representation, and the OSI requires a good flow representation overall, including the vortex breakdown.

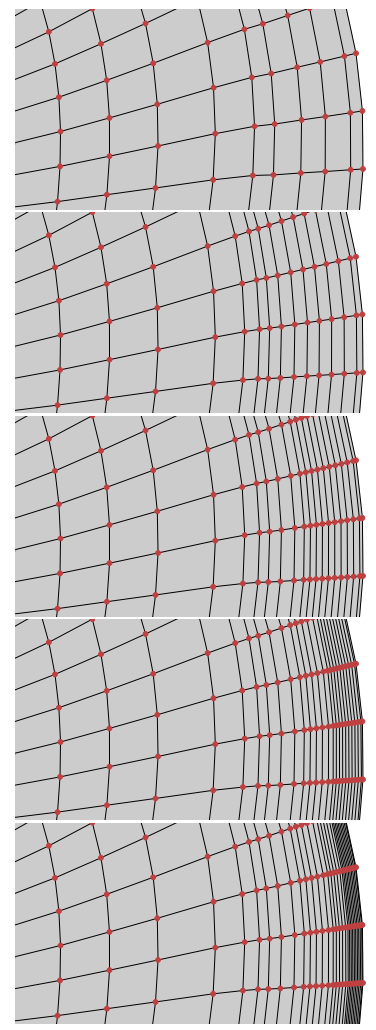

Fig. 21: Patient-specific aorta flow analysis. Control mesh at the inlet. Base Mesh, Refinement Mesh 1, Refinement Mesh 2, Refinement Mesh 3 , and Refinement Mesh 4.

\section{ZSS estimation: patient-specific aorta}

This section is from [20], where more details can be found.

The largest diameter is about $30 \mathrm{~mm}$. The inner-surface mesh is shown in Figure 29. We use Laplace's equation to determine a smooth thickness distribution. We then generate the volume mesh as described in [20], which involves modification of the outer surface and consequently the thickness. The volume mesh is shown in Figure 30. The measured thickness is displayed on the inner-surface mesh in Figure 31. We generate the initial guess following the procedure described in [20].
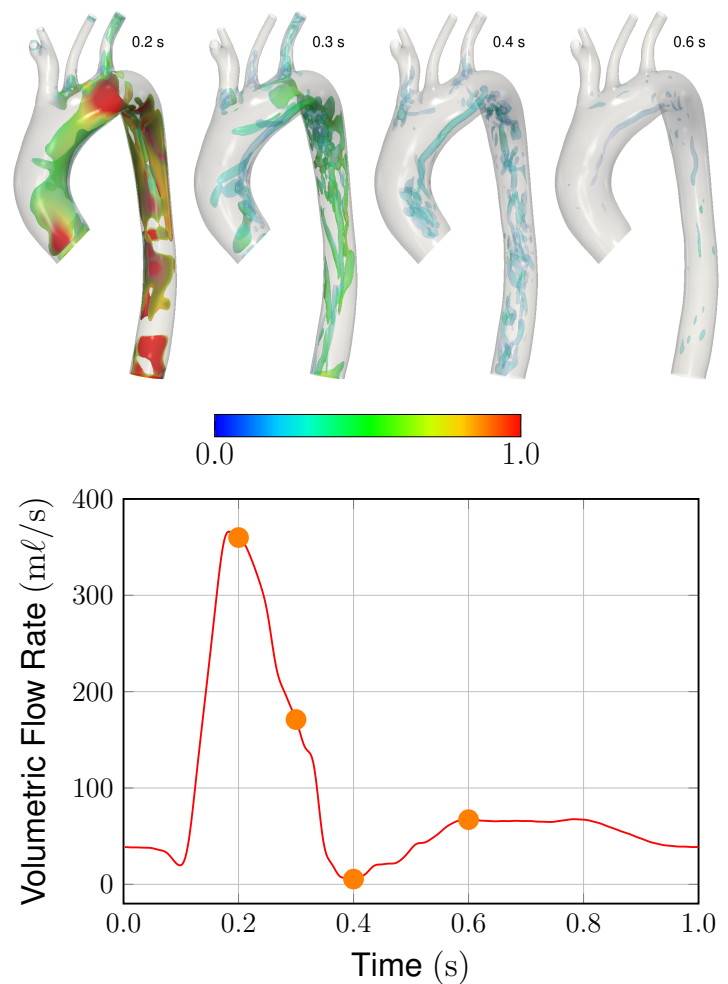

Fig. 22: Patient-specific aorta flow analysis. Mesh refinement study. Computed with Refinement Mesh 4. Isosurfaces corresponding to a positive value of the second invariant of the velocity gradient tensor, colored by the velocity magnitude $(\mathrm{m} / \mathrm{s})(t o p)$. The time instants are shown with circles (bottom). 


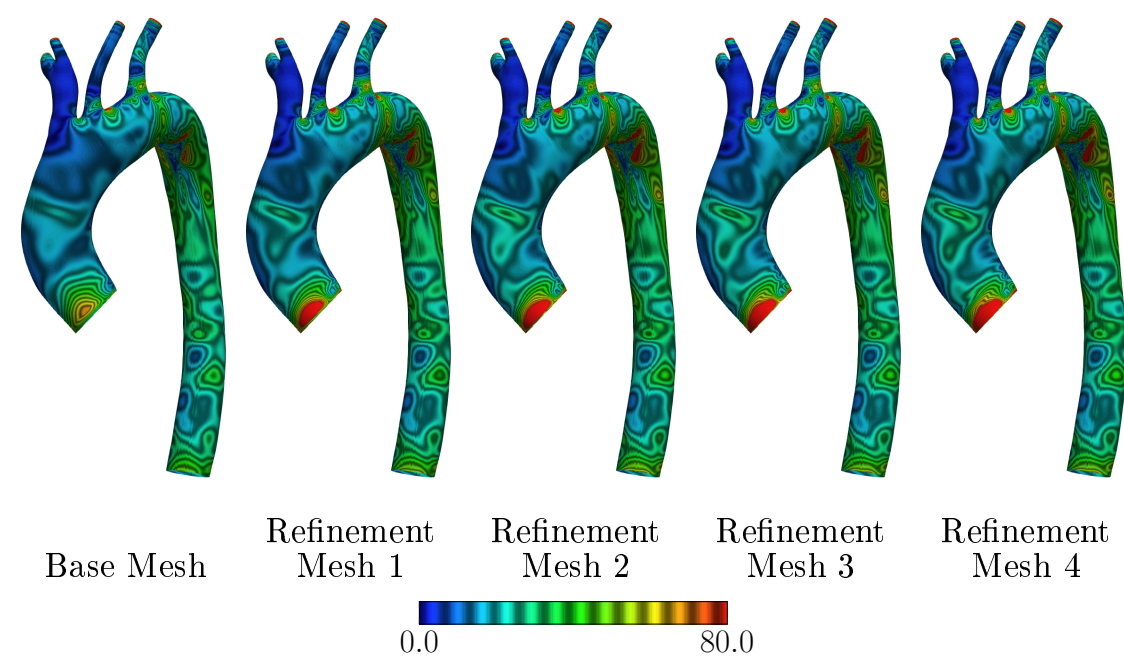

Fig. 23: Patient-specific aorta flow analysis. Mesh refinement study. WSS $\left(\mathrm{dyn} / \mathrm{cm}^{2}\right)$ at the peak flow rate.

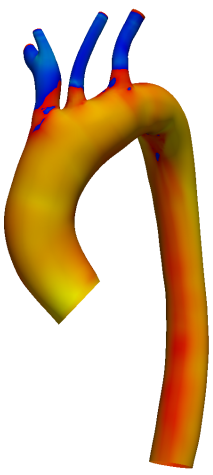

Base Mesh

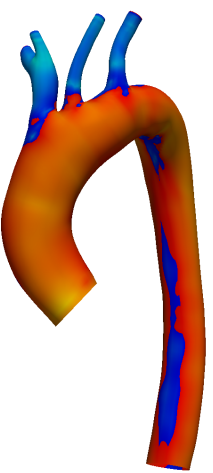

Refinement Mesh 1
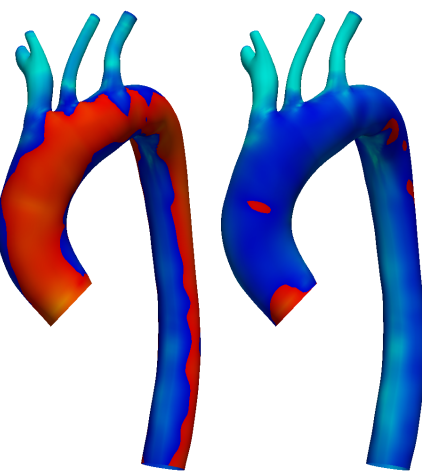

Refinement Mesh 3

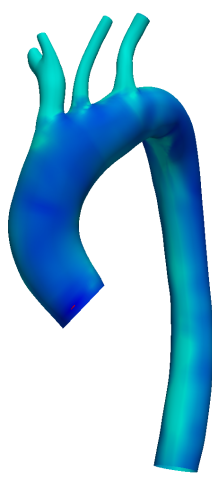

Refinement Mesh 4

Fig. 24: Patient-specific aorta flow analysis. Mesh refinement study. $y^{+}$value for the first-element thickness, based on the WSS computed at the peak flow rate.

Figure 32 shows the IPBZSS, using the EBZSS representation, from the ZSS initial guess and converged ZSS. The stretches are shown in Figures 33 and 34 . Overall, the ZSS initial guess and converged ZSS are very similar. This indicates that reaching the ZSS design targets based on the analytical solution works well.

\section{IMGA computation: patient-specific heart valve design and analysis}

This section is from [146], where more details can be found.

Here we present a novel framework for designing personalized prosthetic heart valves using IMGA-VMS. We parameterize the leaflet geom- 


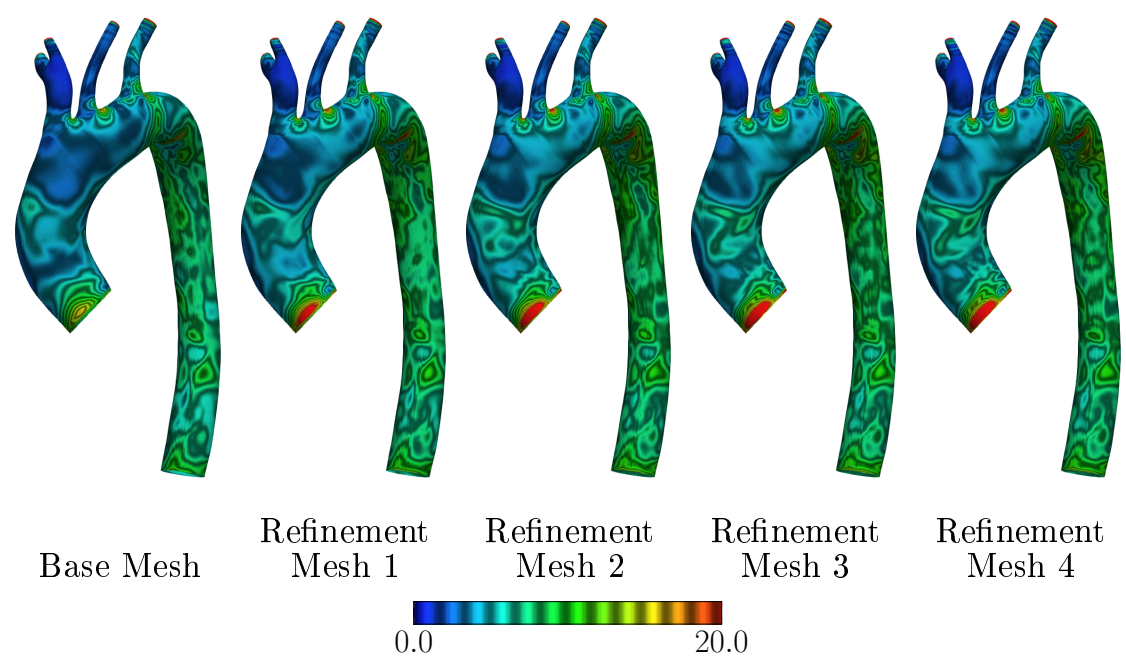

Fig. 25: Patient-specific aorta flow analysis. Mesh refinement study. TAWSS $\left(\mathrm{dyn} / \mathrm{cm}^{2}\right)$.

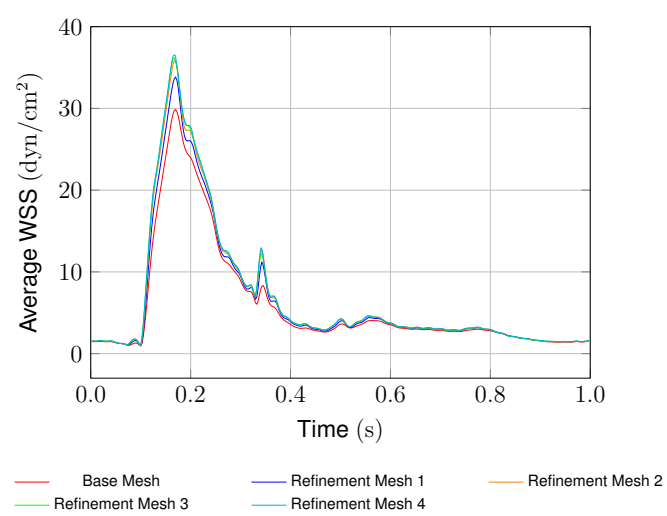

Fig. 26: Patient-specific aorta flow analysis. Mesh refinement study. Spatially-averaged WSS during a cycle.

etry using several key design parameters. This allows for generating various perturbations of the leaflet design for the patient-specific aortic root reconstructed from the medical image data. Each design is analyzed using the IMGAVMS FSI methodology, which allows us to efficiently simulate the coupling of the deforming aortic root, the parametrically designed prosthetic valves, and the surrounding blood flow under physiological conditions. A parametric study is carried out to investigate the influence of the geometry on heart valve performance, indicated by the effective orifice area (EOA) and the coaptation area (CA). Finally, the FSI sim- ulation results of a design that reasonably well balances the EOA and CA are presented.

\subsection{Trivariate NURBS parameterization of the ascending aorta}

To obtain a volumetric parameterization of the artery and lumen, we first construct a trivariate multi-patch NURBS in a regular shape, e.g. a tubular domain, then solve a linear elastostatic, mesh moving problem $[7,171,172]$ for the displacement from this regular domain to a deformed configuration that represents the artery and lumen. However, solving a linear elastostatic problem to obtain the deformed interior mesh is only effective for relatively mild, translation-dominant deformations. For scenarios that involve large deformations, such as the deformation of a straight tubular domain into a curved shape of a patient-specific ascending aorta, the interior elements can become severely distorted. To avoid this, we first obtain a centerline along the axial direction of a patient-specific artery wall surface. Along this centerline, we define a number of cross sections corresponding to the control points of the NURBS artery wall surface in the axial direction. (These cross sections are shown as blue curves in Figure 35a.) At each cross section, we calculate its unit normal 


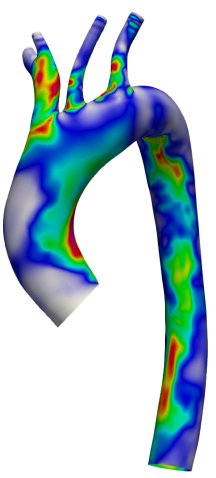

Base Mesh
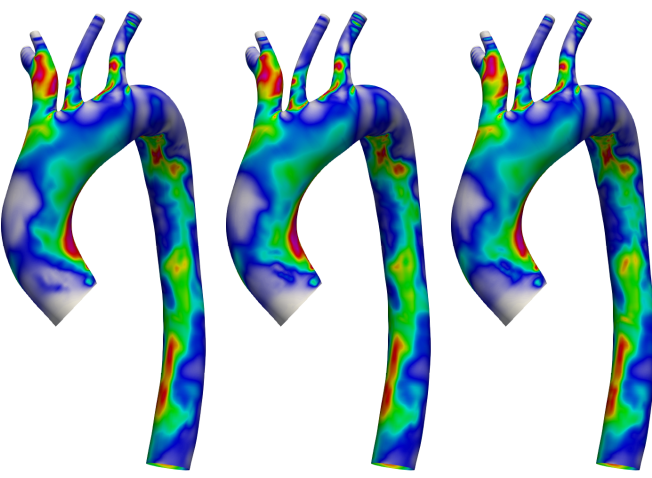

Refinement Mesh 1
Refinement Mesh 2
Refinement Mesh 3

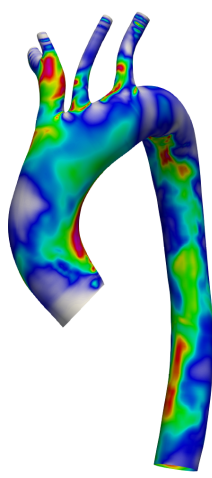

Refinement Mesh 4

Fig. 27: Patient-specific aorta flow analysis. Mesh refinement study. OSI.

vector $\mathbf{n}_{\mathrm{c}}$ and the effective radius $r_{\mathrm{c}}$, which is determined such that the area of a circle calculated using this radius matches the area of the cross section. (A circle corresponding to one of the cross sections is shown in the red curve in Figure 35a.) Finally, using this information, we construct a tubular NURBS surface that has the same control-point and knot-vector topology as the target patient-specific artery wall surface, as shown in Figures 35b and 35c. Another tubular surface corresponding to the lumenal surface is also constructed, using the same cross sections

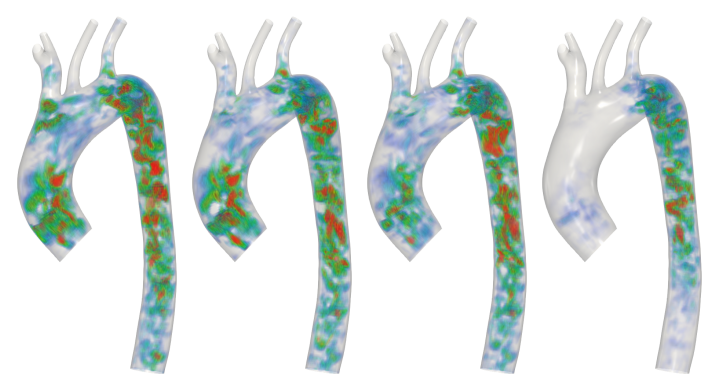

Refinement Refinement Refinement Base Mesh Mesh $1 \quad$ Mesh 2 Mesh 3

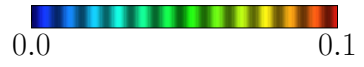

Fig. 28: Patient-specific aorta flow analysis. Mesh refinement study. Velocity difference $\left\|\mathbf{u}_{k}^{h}-\mathbf{u}_{4}^{h}\right\|$ $(\mathrm{m} / \mathrm{s})$ at $0.5 \mathrm{~s}$, where the subscripts indicate Base Mesh and Refinement Mesh $k$. but smaller effective radii coming from the lumenal NURBS surface.

These two tubular NURBS surfaces are used to construct a primitive trivariate multi-patch NURBS that includes the solid and fluid sub-

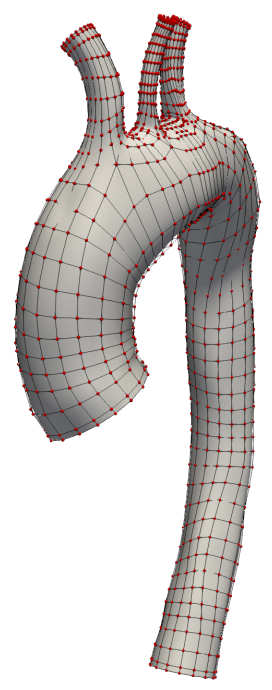

Fig. 29: Patient-specific aorta. Inner-surface mesh, made of cubic and quartic T-splines. Red circles represent the control points. The parts with the quartic T-splines, obtained by order elevation [170], are around the two extraordinary points, each connected to six edges. 
domains, shown in gray and red, respectively, spline space conform to standard fluid-structure in Figure 35d. Basis functions are made $C^{0}$ kinematic constraints while retaining the abilcontinuous at the fluid-solid interface, so that velocity functions defined using the resulting

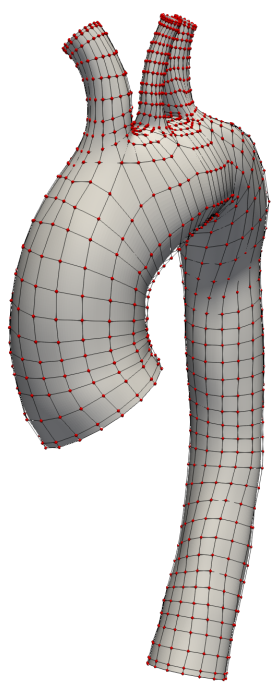
ity to represent non-smooth behavior across the material interface. The resulting volumetric NURBS can then be morphed to match the patient-specific geometry with minimal rotation, so an elastostatic problem can provide an analysis-suitable parameterization. Displacements at the ends of the tube are constrained to

Fig. 30: Patient-specific aorta. Volume mesh, made of cubic and quartic T-splines. Red circles represent the control points.

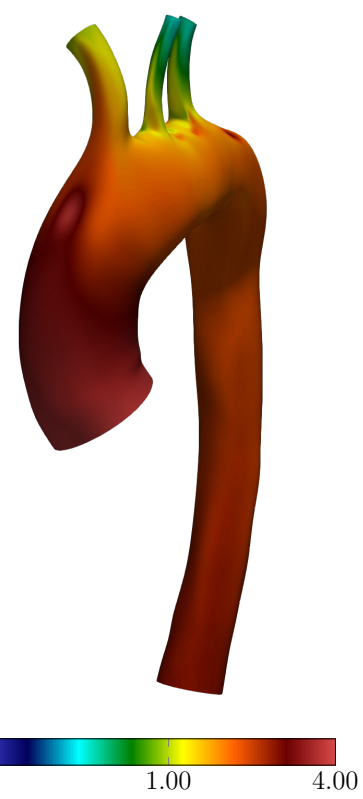

Fig. 31: Patient-specific aorta. Measured thickness (mm).
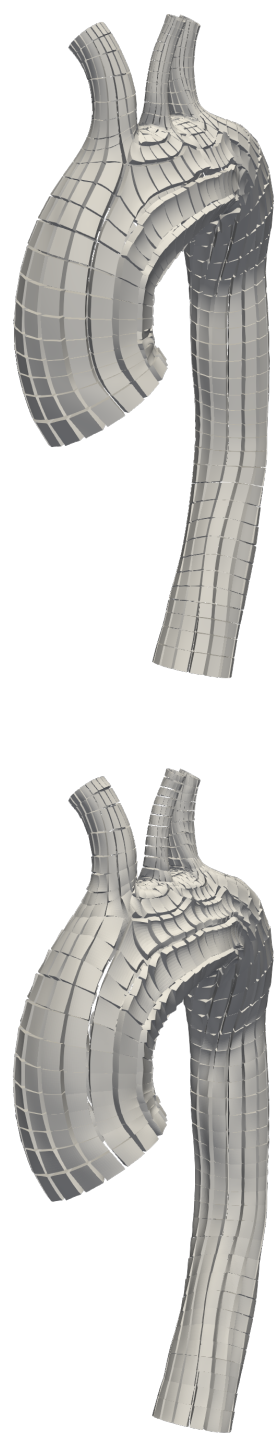

Fig. 32: Patient-specific aorta. The IPBZSS, shown using the EBZSS representation. From the ZSS initial guess (left) and converged ZSS (right). 
remain within their respective cross sections. Finally, we refine the deformed trivariate NURBS for analysis purposes, by inserting knots at desired locations, such as around the sinuses and the flow boundary layers. The final volumetric NURBS discretization of the patient-specific ascending aorta is shown in Figure 35f.
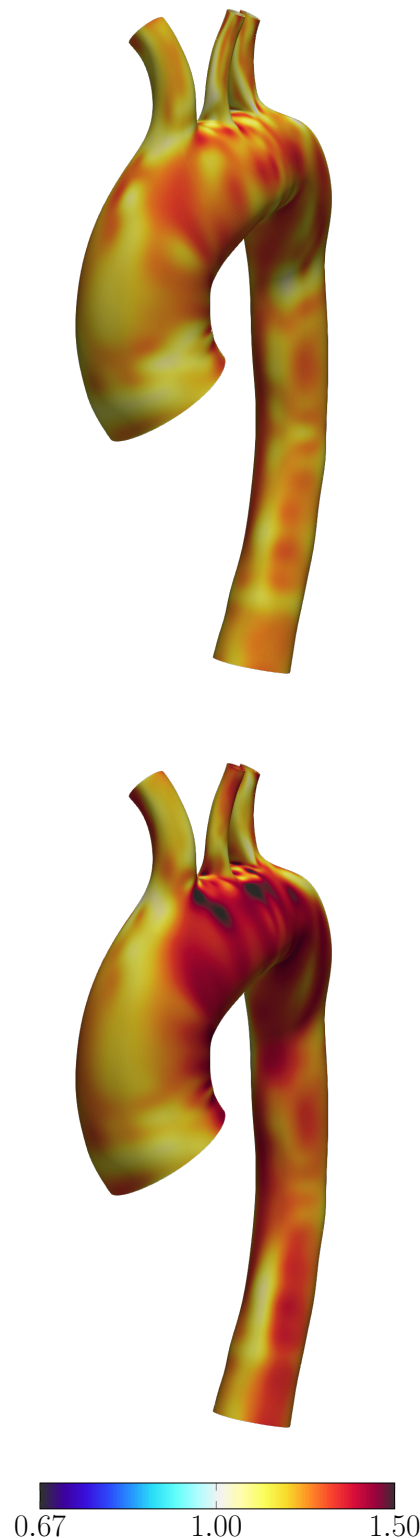

Fig. 33: Patient-specific aorta. Maximum principal stretch. From the ZSS initial guess (left) and converged ZSS (right).

\subsection{Parametric BHV design}

To design effective prosthetic valves for specific patients, we focus specifically on the leaflet geometry and assume that non-leaflet components of stentless valves move with the aortic root and do not affect aortic deformation or flow. Starting from the NURBS surface of a patient-specific
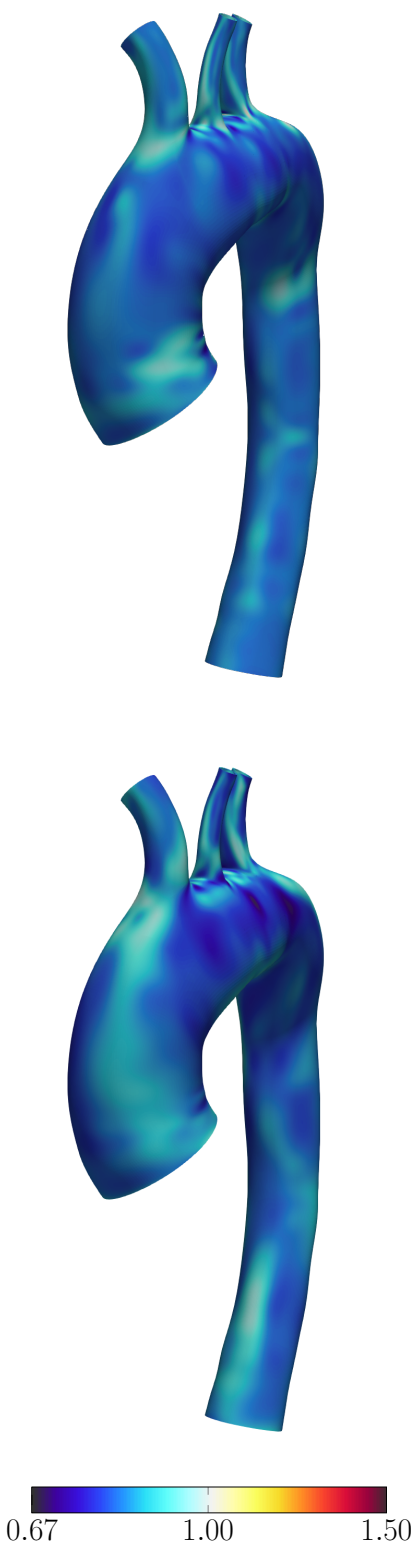

Fig. 34: Patient-specific aorta. Minimum principal stretch. From the ZSS initial guess (left) and converged ZSS (right). 

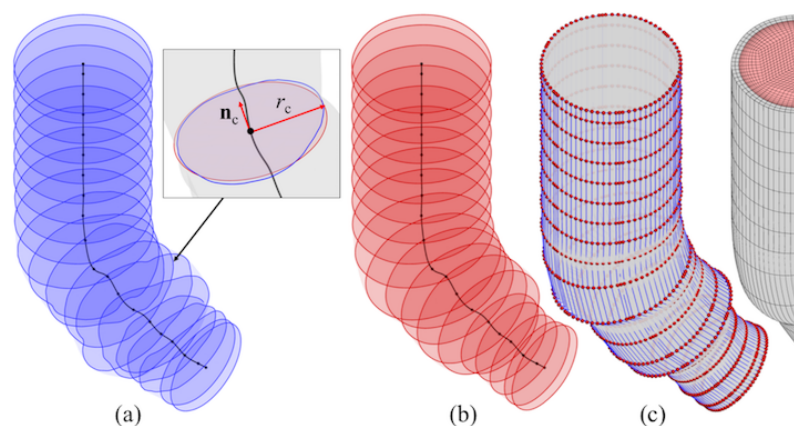

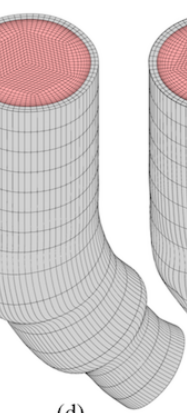

(d)

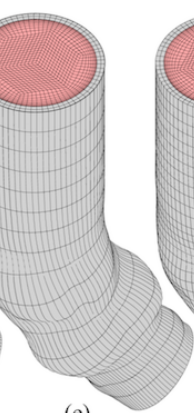

(e)

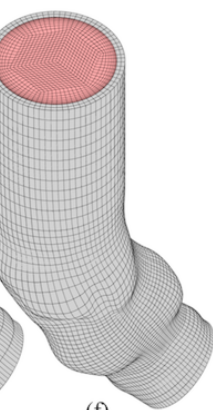

(f)

Fig. 35: The construction of the volumetric NURBS discretization of the blood and the artery wall domains. (a) Cross sections of the artery wall surface. (b) Circular cross sections. (c) NURBS tubular surface and corresponding control points. (d) Primitive volume mesh. (e) Deformed volume mesh. (f) $h$-refined volume mesh.

root, valve leaflets are parametrically designed as follows. We first pick nine "key points" located on the ends of commissure lines and the bottom of the sinuses. The positions of these points are indicated by blue spheres in Figure 36. These define how the leaflets attach to the sinuses. The key points solely depend on the geometry of the patient-specific aortic root and will remain unchanged for different valve designs. We then parameterize families of univariate B-splines defining the free edges and radial "belly curves" of the leaflets. These curves are shown in red and green in Figure 36 . The attachment edges, free edges, and belly curves are then interpolated to obtain smooth bivariate Bspline representations of the leaflets.

Figure 37 shows the details of parameterizing the free-edge curve (red) and the belly-region curve (green). In Figure 37, $\mathbf{p}_{1}, \mathbf{p}_{2}$ and $\mathbf{p}_{3}$ are the key points on the top of the commissure lines

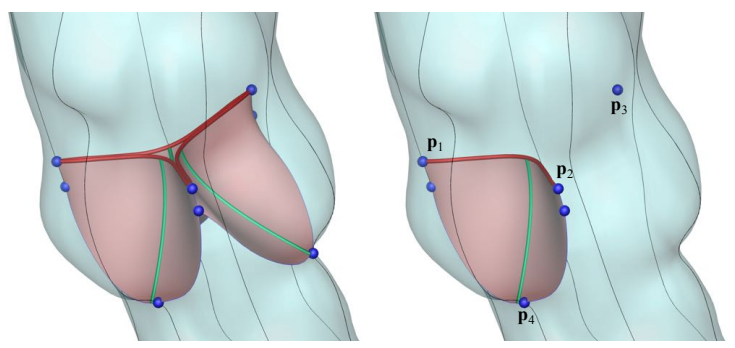

Fig. 36: The key geometric features used to parametrically control the valve designs. The blue key points define the attachment of the valve to the root. The red and green curves are parametrically controlled for valve design. and $\mathbf{p}_{4}$ is the key point on the sinus bottom, as labeled in Figure 36. Points $\mathbf{p}_{1}-\mathbf{p}_{3}$ define a triangle $\Delta \mathbf{p}_{1-3}$, with $\mathbf{p}_{\mathrm{c}}$ being its geometric center. The unit vector pointing from $\mathbf{p}_{\mathrm{c}}$ to $\mathbf{p}_{\mathrm{n}}$ is denoted by $\mathbf{t}_{\mathrm{p}}$, and the unit normal vector of $\Delta \mathbf{p}_{1-3}$ pointing downwards is $\mathbf{n}_{\mathrm{p}}$. We first construct the free edge curve as a univariate quadratic Bspline curve determined by three control points, $\mathbf{p}_{1}, \mathbf{p}_{\mathbf{f}}$, and $\mathbf{p}_{2}$. The location of $\mathbf{p}_{\mathbf{f}}$ is defined by $\mathbf{p}_{\mathrm{f}}=\mathbf{p}_{\mathrm{c}}+x_{1} \mathbf{t}_{\mathrm{p}}+x_{2} \mathbf{n}_{\mathrm{p}}$. By changing $x_{1}$ and $x_{2}$ to control the location of $\mathbf{p}_{\mathrm{f}}$, the curvature and the height of the free edge can be parametrically changed. We then take $\mathbf{p}_{\mathrm{m}}$ as the midpoint of the free edge, the point $\mathbf{p}_{\mathrm{b}}$, and the key point $\mathbf{p}_{4}$ to construct a univariate quadratic Bspline curve (green). The point $\mathbf{p}_{\mathrm{b}}$ is defined by $\mathbf{p}_{\mathrm{b}}=\mathbf{p}_{\mathrm{o}}+x_{3} \mathbf{n}_{\mathrm{p}}$, where $\mathbf{p}_{\mathrm{o}}$ is the projection of $\mathbf{p}_{\mathrm{m}}$ onto $\Delta \mathbf{p}_{1-3}$ along the direction of $\mathbf{n}_{\mathrm{p}}$. Finally, the fixed attachment edges and the parametrically controlled free edge and belly curve are used to construct a cubic B-spline surface with desired parameterization. By choosing $x_{1}$, $x_{2}$ and $x_{3}$ as design variables, we can parametrically change the free edge and belly curve, and therefore change the valve design. This procedure is implemented in an interactive geometry modeling and parametric design platform [173].

\subsection{Application to $\mathrm{BHV}$ design}

To determine an effective BHV design, we first need to identify quantitative measures of its performance. We focus on two quantities of clinical 
interest: to measure the systolic performance, we evaluate the EOA, which indicates how well the valve permits flow in the forward direction. For a quantitative evaluation of the diastolic performance, we measure the $\mathrm{CA}$, which indicates how well the valve seals and prevents flow in the reverse direction. In this section, we study the impact of the design variables $x_{1}, x_{2}$, and $x_{3}$ on our two quantities of interest.

Constitutive parameters in the governing equations are held constant over the design space. Fluid, solid, and shell structure mass densities are set to $1.0 \mathrm{~g} / \mathrm{cm}^{3}$. The parameters of the Fung-type material model for the shell structure are $c_{0}=2.0 \times 10^{6} \mathrm{dyn} / \mathrm{cm}^{2}, c_{1}=2.0 \times 10^{5}$ dyn $/ \mathrm{cm}^{2}$, and $c_{2}=100$. The thickness of the leaflet is set to $0.0386 \mathrm{~cm}$. The bulk and shear modulii for the arterial wall are selected to give a Young's modulus of $10^{7} \mathrm{dyn} / \mathrm{cm}^{2}$ and Poisson's ratio of 0.45 in the small strain limit. The inlet and outlet cross sections are free to slide in their tangential planes and deform radially, but constrained not to move in the orthogonal directions [118]. Mass-proportional damping with constant $C_{\text {damp }}=10^{4} \mathrm{~Hz}$ is used to model the interaction of the artery with the surrounding tissue. The dynamic viscosity of the blood is set to $\mu_{\mathrm{f}}=3 \times 10^{-2} \mathrm{~g} /(\mathrm{cm} \mathrm{s})$.

We apply a physiologically-realistic left ventricular pressure time history as a traction boundary condition at the inflow. The applied pressure signal is periodic, with a period

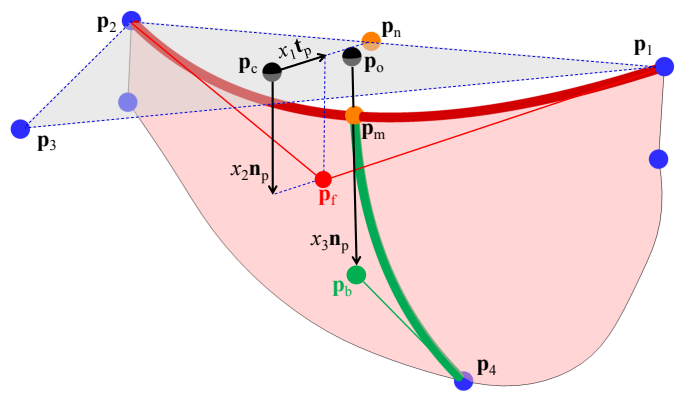

Fig. 37: The parametric control of the valve designs. The key points (blue spheres) are identical to those in the right plot of Figure 36. $x_{1}, x_{2}$, and $x_{3}$ control the location of $\mathbf{P}_{\mathrm{f}}$ and $\mathbf{P}_{\mathrm{b}}$ and thus control the curvature and height of the red free edge, and the curvature of the green belly curve. of $0.86 \mathrm{~s}$ for one cardiac cycle. The traction $-\left(p_{0}+R Q\right) \mathbf{n}_{\mathbf{f}}$ is applied at the outflow for the resistance boundary condition, where $p_{0}$ is a constant physiological pressure level, $R>0$ is a resistance coefficient, and $Q$ is the volumetric flow rate through the outflow. In the present computation, we set $p_{0}=80 \mathrm{mmHg}$ and $R=200$ (dyn $\mathrm{s}) / \mathrm{cm}^{5}$. These values ensure a realistic transvalvular pressure difference of $80 \mathrm{mmHg}$ across a closed valve when $Q=0$, while permitting a flow rate within the normal physiological range and consistent with the flow rate estimated from the medical data (about $310 \mathrm{ml} / \mathrm{s}$ ) during systole. A time step size of $\Delta t=10^{-4} \mathrm{~s}$ is used in all simulations. To obtain the artery wall tissue prestress, we apply the highest left ventricular pressure during systole $(127 \mathrm{mmHg}$ at $t$ $=0.25 \mathrm{~s})$ on the inlet and a resistance boundary condition $\left(p_{0}=80 \mathrm{mmHg}\right.$ and $R=200$ (dyn s) $/ \mathrm{cm}^{5}$ ) on the outlet for the calculation of $\tilde{\mathbf{h}}_{\mathrm{f}}$ in the prestress problem [119].

We perform FSI simulations of each of $\left(x_{1}, x_{2}, x_{3}\right) \in(\{0.05,0.25,0.45\} \mathrm{cm}$, $\{0.1,0.3,0.5\} \mathrm{cm},\{0.5,0.8,1.1,1.4\} \mathrm{cm})$, then calculate the EOA at peak systole and the maximum $\mathrm{CA}$ occurring during ventricular diastole. The simulation results and quantities of interest for each case are reported in [146]. An ideal valve would have both a large EOA and a large CA. However, these two quantities tend to compete with each other: valves that close easily can be more difficult to open and vice versa. In general, the results show that increasing $x_{1}$, which corresponds to decreasing the length of the free edge, decreases EOA and $\mathrm{CA}$ at the same time. Increasing $x_{2}$, which decreases the height of the free edge, may increase EOA slightly but reduces CA significantly. The reduction of $\mathrm{CA}$ due to Increasing $x_{2}$ reduces $\mathrm{CA}$ and causes many designs cannot seal completely. Increasing $x_{3}$, which increases the surface curvature in the leaflet belly region, improves CA but decreases EOA. Finally, the combination of $x_{1}=0.05 \mathrm{~cm}, x_{2}=0.1$ or 0.3 $\mathrm{cm}$, and $x_{3}=0.5$ or $0.8 \mathrm{~cm}$ reliably yields a high EOA between 3.92 and $4.05 \mathrm{~cm}^{2}$, near the upper end of the physiological range of 3.0-4.0 $\mathrm{cm}^{2}$ in healthy adults, and a CA between 3.49 and $4.54 \mathrm{~cm}^{2}$. Among these four cases, $\mathbf{x}^{*}=\left(x_{1}, x_{2}, x_{3}\right)=(0.05 \mathrm{~cm}, 0.1 \mathrm{~cm}, 0.8 \mathrm{~cm})$, 
which has a CA of $4.54 \mathrm{~cm}^{2}$ and EOA of 3.92 $\mathrm{cm}^{2}$, strikes the best compromise between $\mathrm{EOA}$ and $\mathrm{CA}$. The valve geometry of this best-performing design and its EOA and CA from the FSI simulation are shown in Figure 38.

Figure 39 shows several snapshots of the valve deformation and the details of the flow field at several points during the cardiac cycle. The color indicates the fluid velocity magnitude. The visualizations clearly show the instantaneous valve response to the left ventricular pressure. The valve opens with the rising left ventricular pressure in early systole $(0.0-0.20 \mathrm{~s})$, and then stays fully open near peak systole $(0.25-0.27 \mathrm{~s})$, allowing sufficient blood flow to enter the ascending aorta. A quick valve closure is then observed in early diastole $(0.32-0.38 \mathrm{~s})$. This quick closure of the valve minimizes the reverse flow into the left ventricle, as the left ventricular pressure drops rapidly in this period. After that, the valve properly seals, and the flow reaches a near-hydrostatic state $(0.65 \mathrm{~s})$. These features observed during the cardiac cycle characterize a well functioning valve within the objectives considered in this study: a large EOA during systole and a proper CA during diastole. In Figure 40, the models are superposed in the configurations corresponding to the fully-open and fully-closed phases for better visualization of the leaflet-wall coupling results. The deformation of the attach-

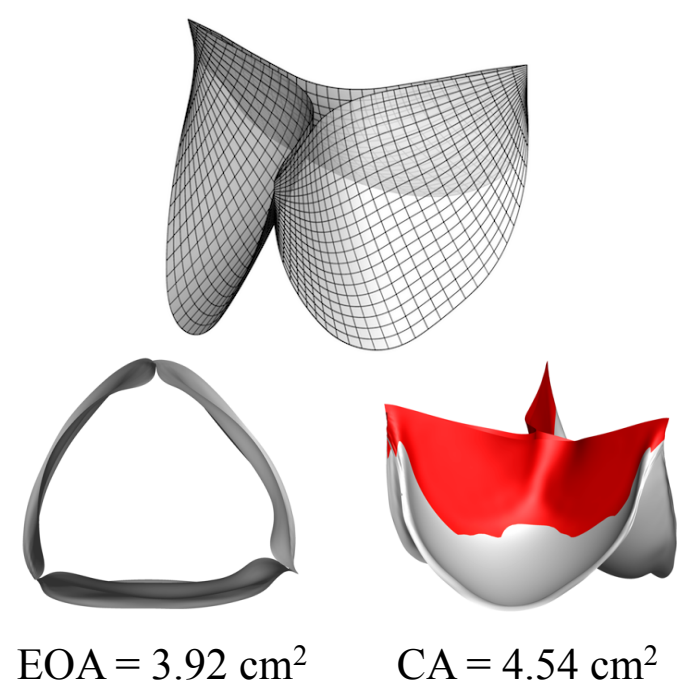

Fig. 38: The best-performing prosthetic valve design and its EOA and CA from the FSI simulation. ment edges can be clearly seen. The expansion and contraction of the arterial wall, as well as its sliding motion between systole and diastole can also be observed. The maximum in-plane principal Green-Lagrange strain (MIPE) evaluated on the aortic side of the leaflet is shown in Figure 41. The figure shows that during opening the strain is concentrated in the belly region of the leaflet, while during closing the highest strain happens near the valve commissure.

\section{Concluding remarks}

We have provided an overview of how the computational challenges faced in cardiovascular flow analysis have been addressed with the STVMS, ALE-VMS, and the IMGA-VMS, which serve the core computational methods, and the special methods used in combination with them. By presenting examples of the challenging computations carried out, we demonstrated that these core and special methods now enable reliable and practical computational analysis in a wide range of patient-specific cases, including cerebral aneurysms, aortas and heart valves. The computational challenges overcome include unsteady flows, complex cardiovascular geometries, MBIs, such as the motion of the heart valve leaflets, contact between moving solid surfaces, such as the contact between the leaflets, and the FSI between the blood and the cardiovascular structure. They also include estimating the ZSS required in the FSI computation. With many of the challenges mostly out of the way, computational cardiovascular flow analysis is now in a better position to provide valuable information to surgeons and medical doctors.

\section{ACKNOWLEDGEMENT}

This work was supported (first author) in part by JST-CREST; Grant-in-Aid for Scientific Research (S) 26220002 from the Ministry of Education, Culture, Sports, Science and Technology of Japan (MEXT); Grant-in-Aid for Scientific Research (A) 18H04100 from Japan Society for the Promotion of Science; and RiceWaseda research agreement. The mathematical model and computational method parts 


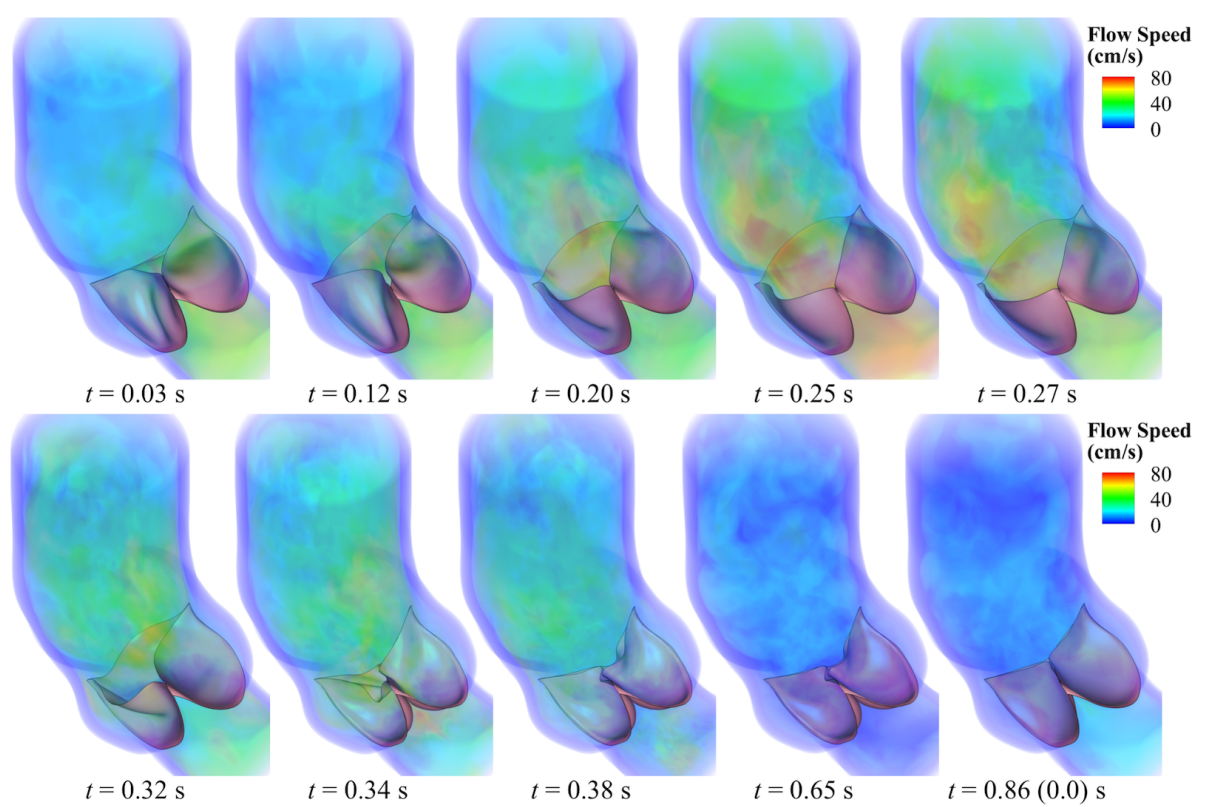

Fig. 39: Volume rendering visualization of the velocity field from our FSI simulation at several points during a cardiac cycle.
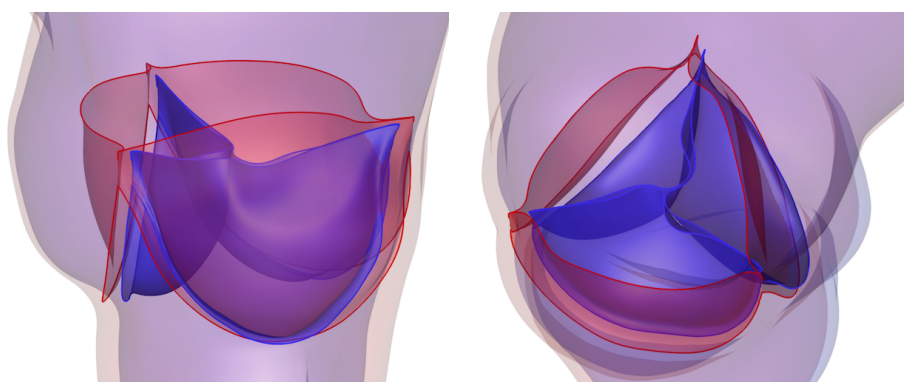

Fig. 40: Relative displacement between fully-open (red) and fully-closed (blue) configurations, showing the effect of leaflet-wall coupling. The deformation of the attachment edges can be clearly seen. The expansion and contraction of the arterial wall as well as its sliding motion between systole (red) and diastole (blue) can also be observed.

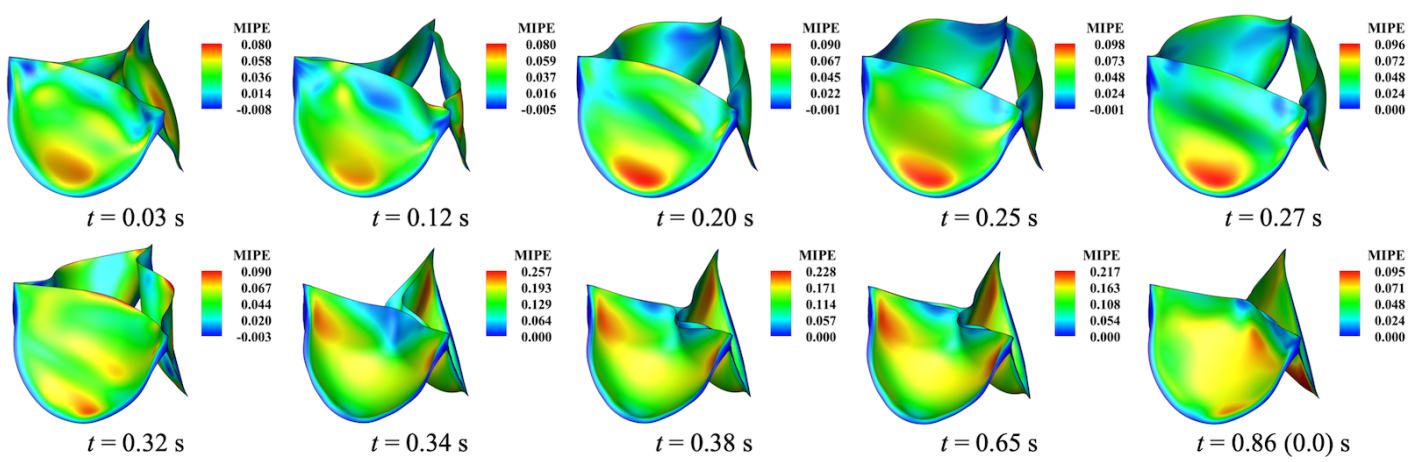

Fig. 41: Deformed valve configuration, colored by the maximum in-plane principal Green-Lagrange strain (MIPE) evaluated on the aortic side of the leaflet. Note the different scale for each time instant. 
of the work were also supported (third author) in part by ARO Grant W911NF-17-1-0046, ARO DURIP Grant W911NF-18-1-0234, and Top Global University Project of Waseda University. The second author was partially supported by NSF Grant 1854436, and the fourth author was partially supported by NIH/NHLBI Grants R01HL129077 and R01HL142504.

\section{References}

[1] Bazilevs, Y., Takizawa, K., \& Tezduyar, T.E. (February 2013). Computational Fluid-Structure Interaction: Methods and Applications. Wiley.

[2] Takizawa, K., Schjodt, K., Puntel, A., Kostov, N., \& Tezduyar, T.E. (2013). Patient-Specific Computational Analysis of the Influence of a Stent on the Unsteady Flow in Cerebral Aneurysms. Computational Mechanics, 51, 1061-1073.

[3] Takizawa, K., Tezduyar, T.E., Uchikawa, H., Terahara, T., Sasaki, T., \& Yoshida, A. (2019). Mesh refinement influence and cardiac-cycle flow periodicity in aorta flow analysis with isogeometric discretization. Computers 89 Fluids, 179, 790-798.

[4] Hsu, M.C., Kamensky, D., Bazilevs, Y., Sacks, M.S., \& Hughes, T.J.R. (2014). Fluid-structure interaction analysis of bioprosthetic heart valves: significance of arterial wall deformation. Computational Mechanics, 54, 1055-1071.

[5] Takizawa, K., Tezduyar, T.E., Terahara, T., \& Sasaki, T. (2017). Heart valve flow computation with the integrated Space-Time VMS, Slip Interface, Topology Change and Isogeometric Discretization methods. Computers \& Fluids, 158, 176-188.

[6] Takizawa, K. \& Tezduyar, T.E. (2011). Multiscale Space-Time Fluid-Structure Interaction Techniques. Computational Mechanics, 48, 247-267.
[7] Bazilevs, Y., Calo, V.M., Hughes, T.J.R., \& Zhang, Y. (2008). Isogeometric fluidstructure interaction: theory, algorithms, and computations. Computational $\mathrm{Me}$ chanics, 43, 3-37.

[8] Kamensky, D., Hsu, M.C., Schillinger, D., Evans, J.A., Aggarwal, A., Bazilevs, Y., Sacks, M.S., \& Hughes, T.J.R. (2015). An immersogeometric variational framework for fluid-structure interaction: Application to bioprosthetic heart valves. Computer Methods in Applied Mechanics and Engineering, 284, 1005-1053.

[9] Takizawa, K., Tezduyar, T.E., Mochizuki, H., Hattori, H., Mei, S., Pan, L., \& Montel, K. (2015). Space-time VMS method for flow computations with slip interfaces (ST-SI). Mathematical Models and Methods in Applied Sciences, 25, 2377-2406.

[10] Takizawa, K., Tezduyar, T.E., Kuraishi, T., Tabata, S., \& Takagi, H. (2016). Computational thermo-fluid analysis of a disk brake. Computational Mechanics, 57, 965977.

[11] Takizawa, K., Tezduyar, T.E., Buscher, A., \& Asada, S. (2014). Space-Time Interface-Tracking with Topology Change (ST-TC). Computational Mechanics, 54, 955-971.

[12] Takizawa, K., Tezduyar, T.E., Buscher, A., \& Asada, S. (2014). Space-Time Fluid Mechanics Computation of Heart Valve Models. Computational Mechanics, 54, 973-986.

[13] Takizawa, K., Henicke, B., Puntel, A., Spielman, T., \& Tezduyar, T.E. (2012). Space-time computational techniques for the aerodynamics of flapping wings. Journal of Applied Mechanics, 79, 010903.

[14] Takizawa, K., Tezduyar, T.E., Otoguro, Y., Terahara, T., Kuraishi, T., \& Hattori, H. (2017). Turbocharger Flow Computations with the Space-Time Isogeometric Analysis (ST-IGA). Computers $\& 5$ Fluids, 142, 15-20. 
[15] Otoguro, Y., Takizawa, K., \& Tezduyar, T.E. (2017). Space-time VMS computational flow analysis with isogeometric discretization and a general-purpose NURBS mesh generation method. Computers 83 Fluids, 158, 189-200.

[16] Otoguro, Y., Takizawa, K., \& Tezduyar, T.E. (2018). A General-Purpose NURBS Mesh Generation Method for Complex Geometries. In Tezduyar, T.E. (editor), Frontiers in Computational Fluid-Structure Interaction and Flow Simulation: Research from Lead Investigators under Forty - 2018, Modeling and Simulation in Science, Engineering and Technology, Springer, 399-434.

[17] Takizawa, K., Takagi, H., Tezduyar, T.E., \& Torii, R. (2014). Estimation of ElementBased Zero-Stress State for Arterial FSI Computations. Computational Mechanics, 54, 895-910.

[18] Takizawa, K., Tezduyar, T.E., \& Sasaki, T. (2018). Estimation of element-based zero-stress state in arterial FSI computations with isogeometric wall discretization. In Wriggers, P. \& Lenarz, T. (editors), Biomedical Technology: Modeling, Experiments and Simulation, Lecture Notes in Applied and Computational Mechanics, Springer, 101-122.

[19] Sasaki, T., Takizawa, K., \& Tezduyar, T.E. (2019). Aorta Zero-Stress State Modeling with T-Spline Discretization. Computational Mechanics, 63, 1315-1331.

[20] Sasaki, T., Takizawa, K., \& Tezduyar, T.E. (February 2019). MedicalImage-Based Aorta Modeling with Zero-Stress-State Estimation, Computational Mechanics, published online, DOI: 10.1007/s00466-019-01669-4.

[21] Bazilevs, Y. \& Hughes, T.J.R. (2007). Weak imposition of Dirichlet boundary conditions in fluid mechanics. Computers and Fluids, 36, 12-26.

[22] Bazilevs, Y., Michler, C., Calo, V.M., \& Hughes, T.J.R. (2010). Isogeometric variational multiscale modeling of wallbounded turbulent flows with weakly enforced boundary conditions on unstretched meshes. Computer Methods in Applied Mechanics and Engineering, 199, 780-790.

[23] Hsu, M.C., Akkerman, I., \& Bazilevs, Y. (2012). Wind turbine aerodynamics using ALE-VMS: Validation and role of weakly enforced boundary conditions. Computational Mechanics, 50, 499-511.

[24] Bazilevs, Y. \& Hughes, T.J.R. (2008). NURBS-based isogeometric analysis for the computation of flows about rotating components. Computational Mechanics, 43, 143-150.

[25] Hsu, M.C. \& Bazilevs, Y. (2012). Fluidstructure interaction modeling of wind turbines: simulating the full machine. Computational Mechanics, 50, 821-833.

[26] Moghadam, M.E., Bazilevs, Y., Hsia, T.Y., Vignon-Clementel, I.E., Marsden, A.L., \& of Congenital Hearts Alliance (MOCHA), M. (2011). A comparison of outlet boundary treatments for prevention of backflow divergence with relevance to blood flow simulations. Computational Mechanics, 48, 277-291.

[27] Tezduyar, T.E. (1992). Stabilized Finite Element Formulations for Incompressible Flow Computations. Advances in Applied Mechanics, 28, 1-44.

[28] Tezduyar, T.E. (2003). Computation of Moving Boundaries and Interfaces and Stabilization Parameters. International Journal for Numerical Methods in Fluids, $43,555-575$.

[29] Tezduyar, T.E. \& Sathe, S. (2007). Modeling of Fluid-Structure Interactions with the Space-Time Finite Elements: Solution Techniques. International Journal for $\mathrm{Nu}$ merical Methods in Fluids, 54, 855-900.

[30] Brooks, A.N. \& Hughes, T.J.R. (1982). Streamline Upwind/Petrov-Galerkin Formulations for Convection Dominated Flows with Particular Emphasis on the 
Incompressible Navier-Stokes Equations. Computer Methods in Applied Mechanics and Engineering, 32, 199-259.

[31] Hughes, T.J.R. (1995). Multiscale Phenomena: Green's Functions, The Dirichlet-to-Neumann Formulation, Subgrid Scale Models, Bubbles, and the Origins of Stabilized Methods. Computer Methods in Applied Mechanics and Engineering, 127, 387-401.

[32] Hughes, T.J.R., Oberai, A.A., \& Mazzei, L. (2001). Large Eddy Simulation of Turbulent Channel Flows by the Variational Multiscale Method. Physics of Fluids, 13, 1784-1799.

[33] Bazilevs, Y., Calo, V.M., Cottrell, J.A., Hughes, T.J.R., Reali, A., \& Scovazzi, G. (2007). Variational multiscale residualbased turbulence modeling for large eddy simulation of incompressible flows. Computer Methods in Applied Mechanics and Engineering, 197, 173-201.

[34] Bazilevs, Y. \& Akkerman, I. (2010). Large eddy simulation of turbulent TaylorCouette flow using isogeometric analysis and the residual-based variational multiscale method. Journal of Computational Physics, 229, 3402-3414.

[35] Takizawa, K. \& Tezduyar, T.E. (2012). Space-Time Fluid-Structure Interaction Methods. Mathematical Models and Methods in Applied Sciences, 22(supp02), 1230001 .

[36] Tezduyar, T.E. \& Takizawa, K. (2019). Space-time computations in practical engineering applications: A summary of the 25-year history. Computational Mechanics, 63, 747-753.

[37] Takizawa, K. \& Tezduyar, T.E. (2012). Computational Methods for Parachute Fluid-Structure Interactions. Archives of Computational Methods in Engineering, 19, 125-169.

[38] Takizawa, K., Fritze, M., Montes, D., Spielman, T., \& Tezduyar, T.E. (2012). Fluid-structure interaction modeling of ringsail parachutes with disreefing and modified geometric porosity. Computational Mechanics, 50, 835-854.

[39] Takizawa, K., Tezduyar, T.E., Boben, J., Kostov, N., Boswell, C., \& Buscher, A. (2013). Fluid-structure interaction modeling of clusters of spacecraft parachutes with modified geometric porosity. Computational Mechanics, 52, 1351-1364.

[40] Takizawa, K., Tezduyar, T.E., Boswell, C., Tsutsui, Y., \& Montel, K. (2015). Special Methods for Aerodynamic-Moment Calculations from Parachute FSI Modeling. Computational Mechanics, 55, 1059-1069.

[41] Takizawa, K., Montes, D., Fritze, M., McIntyre, S., Boben, J., \& Tezduyar, T.E. (2013). Methods for FSI modeling of spacecraft parachute dynamics and cover separation. Mathematical Models and Methods in Applied Sciences, 23, 307338.

[42] Takizawa, K., Tezduyar, T.E., Boswell, C., Kolesar, R., \& Montel, K. (2014). FSI Modeling of the Reefed Stages and Disreefing of the Orion Spacecraft Parachutes. Computational Mechanics, 54, 1203-1220.

[43] Takizawa, K., Tezduyar, T.E., Kolesar, R., Boswell, C., Kanai, T., \& Montel, K. (2014). Multiscale Methods for Gore Curvature Calculations from FSI Modeling of Spacecraft Parachutes. Computational Mechanics, 54, 1461-1476.

[44] Takizawa, K., Tezduyar, T.E., \& Kolesar, R. (2015). FSI Modeling of the Orion Spacecraft Drogue Parachutes. Computational Mechanics, 55, 1167-1179.

[45] Bazilevs, Y., Hsu, M.C., Akkerman, I., Wright, S., Takizawa, K., Henicke, B., Spielman, T., \& Tezduyar, T.E. (2011). 3D Simulation of Wind Turbine Rotors at Full Scale. Part I: Geometry Modeling and Aerodynamics. International Journal for Numerical Methods in Fluids, 65, 207-235.

[46] Takizawa, K., Henicke, B., Tezduyar, T.E., Hsu, M.C., \& Bazilevs, Y. (2011). Stabilized Space-Time Computation of 
Wind-Turbine Rotor Aerodynamics. Computational Mechanics, 48, 333-344.

[47] Takizawa, K., Henicke, B., Montes, D., Tezduyar, T.E., Hsu, M.C., \& Bazilevs, Y. (2011). Numerical-Performance Studies for the Stabilized Space-Time Computation of Wind-Turbine Rotor Aerodynamics. Computational Mechanics, 48, 647657.

[48] Takizawa, K., Tezduyar, T.E., McIntyre, S., Kostov, N., Kolesar, R., \& Habluetzel, C. (2014). Space-time VMS computation of wind-turbine rotor and tower aerodynamics. Computational Mechanics, 53, 115.

[49] Takizawa, K., Bazilevs, Y., Tezduyar, T.E., Hsu, M.C., Øiseth, O., Mathisen, K.M., Kostov, N., \& McIntyre, S. (2014). Engineering Analysis and Design with ALE-VMS and Space-Time Methods. Archives of Computational Methods in Engineering, 21, 481-508.

[50] Takizawa, K. (2014). Computational Engineering Analysis with the New-Generation Space-Time Methods. Computational Mechanics, 54, 193-211.

[51] Bazilevs, Y., Takizawa, K., Tezduyar, T.E., Hsu, M.C., Kostov, N., \& McIntyre, S. (2014). Aerodynamic and FSI Analysis of Wind Turbines with the ALE-VMS and ST-VMS Methods. Archives of Computational Methods in Engineering, 21, 359398.

[52] Korobenko, A., Bazilevs, Y., Takizawa, K., \& Tezduyar, T.E. (2018). Recent Advances in ALE-VMS and ST-VMS Computational Aerodynamic and FSI Analysis of Wind Turbines. In Tezduyar, T.E. (editor), Frontiers in Computational FluidStructure Interaction and Flow Simulation: Research from Lead Investigators under Forty - 2018, Modeling and Simulation in Science, Engineering and Technology, Springer, 253-336.

[53] Korobenko, A., Bazilevs, Y., Takizawa, K., \& Tezduyar, T.E. (September 2018).
Computer modeling of wind turbines: 1 . ALE-VMS and ST-VMS aerodynamic and FSI analysis, Archives of Computational Methods in Engineering, to appear, DOI: 10.1007/s11831-018-9292-1.

[54] Takizawa, K., Henicke, B., Puntel, A., Kostov, N., \& Tezduyar, T.E. (2012). Space-Time Techniques for Computational Aerodynamics Modeling of Flapping Wings of an Actual Locust. Computational Mechanics, 50, 743-760.

[55] Takizawa, K., Henicke, B., Puntel, A., Kostov, N., \& Tezduyar, T.E. (2013). Computer Modeling Techniques for Flapping-Wing Aerodynamics of a Locust. Computers \&5 Fluids, 85, 125-134.

[56] Takizawa, K., Kostov, N., Puntel, A., Henicke, B., \& Tezduyar, T.E. (2012). Space-time computational analysis of bioinspired flapping-wing aerodynamics of a micro aerial vehicle. Computational $\mathrm{Me}$ chanics, 50, 761-778.

[57] Takizawa, K., Tezduyar, T.E., \& Kostov, N. (2014). Sequentially-coupled spacetime FSI analysis of bio-inspired flappingwing aerodynamics of an MAV. Computational Mechanics, 54, 213-233.

[58] Takizawa, K., Tezduyar, T.E., \& Buscher, A. (2015). Space-Time Computational Analysis of MAV Flapping-Wing Aerodynamics with Wing Clapping. Computational Mechanics, 55, 1131-1141.

[59] Takizawa, K., Bazilevs, Y., Tezduyar, T.E., Long, C.C., Marsden, A.L., \& Schjodt, K. (2014). ST and ALE-VMS Methods for Patient-Specific Cardiovascular Fluid Mechanics Modeling. Mathematical Models and Methods in Applied Sciences, 24, 2437-2486.

[60] Takizawa, K., Schjodt, K., Puntel, A., Kostov, N., \& Tezduyar, T.E. (2012). Patient-specific computer modeling of blood flow in cerebral arteries with aneurysm and stent. Computational $\mathrm{Me}$ chanics, 50, 675-686. 
[61] Suito, H., Takizawa, K., Huynh, V.Q.H., Sze, D., \& Ueda, T. (2014). FSI analysis of the blood flow and geometrical characteristics in the thoracic aorta. Computational Mechanics, 54, 1035-1045.

[62] Suito, H., Takizawa, K., Huynh, V.Q.H., Sze, D., Ueda, T., \& Tezduyar, T.E. (2016). A geometrical-characteristics study in patient-specific FSI analysis of blood flow in the thoracic aorta. In Bazilevs, Y. \& Takizawa, K. (editors), Advances in Computational Fluid-Structure Interaction and Flow Simulation: New Methods and Challenging Computations, Modeling and Simulation in Science, Engineering and Technology, Springer, 379-386.

[63] Takizawa, K., Tezduyar, T.E., Uchikawa, H., Terahara, T., Sasaki, T., Shiozaki, K., Yoshida, A., Komiya, K., \& Inoue, G. (2018). Aorta Flow Analysis and Heart Valve Flow and Structure Analysis. In Tezduyar, T.E. (editor), Frontiers in Computational Fluid-Structure Interaction and Flow Simulation: Research from Lead Investigators under Forty - 2018, Modeling and Simulation in Science, Engineering and Technology, Springer, 29-89.

[64] Takizawa, K. \& Tezduyar, T.E. (2016). New directions in space-time computational methods. In Bazilevs, Y. \& Takizawa, K. (editors), Advances in Computational Fluid-Structure Interaction and Flow Simulation: New Methods and Challenging Computations, Modeling and Simulation in Science, Engineering and Technology, Springer, 159-178.

[65] Takizawa, K., Tezduyar, T.E., Terahara, T., \& Sasaki, T. (2018). Heart valve flow computation with the Space-Time Slip Interface Topology Change (ST-SI-TC) method and Isogeometric Analysis (IGA). In Wriggers, P. \& Lenarz, T. (editors), Biomedical Technology: Modeling, Experiments and Simulation, Lecture Notes in Applied and Computational Mechanics, Springer, 77-99.
[66] Takizawa, K., Montes, D., McIntyre, S., \& Tezduyar, T.E. (2013). Space-Time VMS Methods for Modeling of Incompressible Flows at High Reynolds Numbers. Mathematical Models and Methods in Applied Sciences, 23, 223-248.

[67] Takizawa, K., Tezduyar, T.E., \& Kuraishi, T. (2015). Multiscale ST Methods for Thermo-Fluid Analysis of a Ground Vehicle and its Tires. Mathematical Models and Methods in Applied Sciences, 25, 2227-2255.

[68] Takizawa, K., Tezduyar, T.E., \& Hattori, H. (2017). Computational Analysis of Flow-Driven String Dynamics in Turbomachinery. Computers \& Fluids, 142, 109117.

[69] Komiya, K., Kanai, T., Otoguro, Y., Kaneko, M., Hirota, K., Zhang, Y., Takizawa, K., Tezduyar, T.E., Nohmi, M., Tsuneda, T., Kawai, M., \& Isono, M. (2019). Computational analysis of flowdriven string dynamics in a pump and residence time calculation. IOP conference series earth and environmental science, 240 , 062014 .

[70] Kanai, T., Takizawa, K., Tezduyar, T.E., Komiya, K., Kaneko, M., Hirota, K., Nohmi, M., Tsuneda, T., Kawai, M., \& Isono, M. (February 2019). Methods for Computation of Flow-Driven String Dynamics in a Pump and Residence Time, Mathematical Models and Methods in Applied Sciences, published online, DOI: 10.1142/S021820251941001X.

[71] Otoguro, Y., Takizawa, K., Tezduyar, T.E., Nagaoka, K., \& Mei, S. (2019). Turbocharger turbine and exhaust manifold flow computation with the Space-Time Variational Multiscale Method and Isogeometric Analysis. Computers \& Fluids, 179, 764-776.

[72] Takizawa, K., Tezduyar, T.E., Asada, S., \& Kuraishi, T. (2016). Space-Time Method for Flow Computations with Slip Interfaces and Topology Changes (ST-SITC). Computers \& Fluids, 141, 124-134. 
[73] Kuraishi, T., Takizawa, K., \& Tezduyar, T.E. (2018). Space-Time Computational Analysis of Tire Aerodynamics with Actual Geometry, Road Contact and Tire Deformation. In Tezduyar, T.E. (editor), Frontiers in Computational FluidStructure Interaction and Flow Simulation: Research from Lead Investigators under Forty - 2018, Modeling and Simulation in Science, Engineering and Technology, Springer, 337-376.

[74] Kuraishi, T., Takizawa, K., \& Tezduyar, T.E. (2019). Tire Aerodynamics with Actual Tire Geometry, Road Contact and Tire Deformation. Computational Mechanics, 63, 1165-1185.

[75] Kuraishi, T., Takizawa, K., \& Tezduyar, T.E. (February 2019). SpaceTime Isogeometric Flow Analysis with Built-in Reynolds-Equation Limit, Mathematical Models and Methods in Applied Sciences, published online, DOI: 10.1142/S0218202519410021.

[76] Takizawa, K., Tezduyar, T.E., \& Terahara, T. (2016). Ram-Air Parachute Structural and Fluid Mechanics Computations with the Space-Time Isogeometric Analysis (ST-IGA). Computers \& Fluids, 141, 191-200.

[77] Takizawa, K., Tezduyar, T.E., \& Kanai, T. (2017). Porosity models and computational methods for compressible-flow aerodynamics of parachutes with geometric porosity. Mathematical Models and Methods in Applied Sciences, 27, 771-806.

[78] Kanai, T., Takizawa, K., Tezduyar, T.E., Tanaka, T., \& Hartmann, A. (2019). Compressible-Flow Geometric-Porosity Modeling and Spacecraft Parachute Computation with Isogeometric Discretization. Computational Mechanics, 63, 301-321.

[79] Torii, R., Oshima, M., Kobayashi, T., Takagi, K., \& Tezduyar, T.E. (2004). Computation of Cardiovascular Fluid-Structure Interactions with the DSD/SST Method. In Proceedings of the 6th World Congress on Computational Mechanics (CD-ROM), Beijing, China.
[80] Torii, R., Oshima, M., Kobayashi, T., Takagi, K., \& Tezduyar, T.E. (2004). Influence of Wall Elasticity on Image-Based Blood Flow Simulations. Transactions of the Japan Society of Mechanical Engineers Series A, 70, 1224-1231, in Japanese.

[81] Torii, R., Oshima, M., Kobayashi, T., Takagi, K., \& Tezduyar, T.E. (2006). Computer Modeling of Cardiovascular Fluid-Structure Interactions with the Deforming-Spatial-Domain/Stabilized Space-Time Formulation. Computer Methods in Applied Mechanics and Engineering, 195, 1885-1895.

[82] Torii, R., Oshima, M., Kobayashi, T., Takagi, K., \& Tezduyar, T.E. (2006). Fluid-Structure Interaction Modeling of Aneurysmal Conditions with High and Normal Blood Pressures. Computational Mechanics, 38, 482-490.

[83] Tezduyar, T.E., Sathe, S., Cragin, T., Nanna, B., Conklin, B.S., Pausewang, J., \& Schwaab, M. (2007). Modeling of FluidStructure Interactions with the SpaceTime Finite Elements: Arterial Fluid Mechanics. International Journal for Numerical Methods in Fluids, 54, 901-922.

[84] Tezduyar, T.E., Sathe, S., Schwaab, M., \& Conklin, B.S. (2008). Arterial Fluid Mechanics Modeling with the Stabilized Space-Time Fluid-Structure Interaction Technique. International Journal for $\mathrm{Nu}$ merical Methods in Fluids, 57, 601-629.

[85] Tezduyar, T.E., Schwaab, M., \& Sathe, S. (2009). Sequentially-Coupled Arterial Fluid-Structure Interaction (SCAFSI) technique. Computer Methods in Applied Mechanics and Engineering, 198, 35243533.

[86] Takizawa, K., Christopher, J., Tezduyar, T.E., \& Sathe, S. (2010). Space-Time Finite Element Computation of Arterial Fluid-Structure Interactions with PatientSpecific Data. International Journal for Numerical Methods in Biomedical Engineering, 26, 101-116. 
[87] Tezduyar, T.E., Takizawa, K., Moorman, C., Wright, S., \& Christopher, J. (2010). Multiscale Sequentially-Coupled Arterial FSI Technique. Computational Mechanics, 46, 17-29.

[88] Takizawa, K., Moorman, C., Wright, S., Christopher, J., \& Tezduyar, T.E. (2010). Wall Shear Stress Calculations in SpaceTime Finite Element Computation of Arterial Fluid-Structure Interactions. Computational Mechanics, 46, 31-41.

[89] Takizawa, K., Moorman, C., Wright, S., Purdue, J., McPhail, T., Chen, P.R., Warren, J., \& Tezduyar, T.E. (2011). PatientSpecific Arterial Fluid-Structure Interaction Modeling of Cerebral Aneurysms. International Journal for Numerical Methods in Fluids, 65, 308-323.

[90] Tezduyar, T.E., Takizawa, K., Brummer, T., \& Chen, P.R. (2011). Space-Time Fluid-Structure Interaction Modeling of Patient-Specific Cerebral Aneurysms. International Journal for Numerical Methods in Biomedical Engineering, 27, 16651710.

[91] Takizawa, K., Brummer, T., Tezduyar, T.E., \& Chen, P.R. (2012). A comparative study based on patient-specific fluidstructure interaction modeling of cerebral aneurysms. Journal of Applied Mechanics, 79, 010908.

[92] Takizawa, K., Schjodt, K., Puntel, A., Kostov, N., \& Tezduyar, T.E. (2013). Patient-Specific Computational Fluid Mechanics of Cerebral Arteries with Aneurysm and Stent. In Li, S. \& Qian, D. (editors), Multiscale Simulations and Mechanics of Biological Materials, chapter 7, Wiley, 119-147.

[93] Takizawa, K., Bazilevs, Y., Tezduyar, T.E., Long, C.C., Marsden, A.L., \& Schjodt, K. (2014). Patient-Specific Cardiovascular Fluid Mechanics Analysis with the ST and ALE-VMS Methods. In Idelsohn, S.R. (editor), Numerical Simulations of Coupled Problems in Engineering, volume 33 of Computational Methods in Ap- plied Sciences, chapter 4, Springer, 71102.

[94] Takizawa, K., Torii, R., Takagi, H., Tezduyar, T.E., \& Xu, X.Y. (2014). Coronary arterial dynamics computation with medical-image-based time-dependent anatomical models and element-based zero-stress state estimates. Computational Mechanics, 54, 1047-1053.

[95] Takizawa, K., Bazilevs, Y., \& Tezduyar, T.E. (2012). Space-Time and ALE-VMS Techniques for Patient-Specific Cardiovascular Fluid-Structure Interaction Modeling. Archives of Computational Methods in Engineering, 19, 171-225.

[96] Bazilevs, Y., Hsu, M.C., Takizawa, K., \& Tezduyar, T.E. (2012). ALE-VMS and ST-VMS Methods for Computer Modeling of Wind-Turbine Rotor Aerodynamics and Fluid-Structure Interaction. Mathematical Models and Methods in Applied Sciences, 22(supp02), 1230002.

[97] Bazilevs, Y., Takizawa, K., \& Tezduyar, T.E. (2013). Challenges and Directions in Computational Fluid-Structure Interaction. Mathematical Models and Methods in Applied Sciences, 23, 215-221.

[98] Bazilevs, Y., Takizawa, K., \& Tezduyar, T.E. (2015). New Directions and Challenging Computations in Fluid Dynamics Modeling with Stabilized and Multiscale Methods. Mathematical Models and Methods in Applied Sciences, 25, 2217-2226.

[99] Bazilevs, Y., Takizawa, K., \& Tezduyar, T.E. (February 2019). Computational Analysis Methods for Complex Unsteady Flow Problems, Mathematical Models and Methods in Applied Sciences, published online, DOI: 10.1142/S0218202519020020.

[100] Hughes, T.J.R., Liu, W.K., \& Zimmermann, T.K. (1981). Lagrangian-Eulerian finite element formulation for incompressible viscous flows. Computer Methods in Applied Mechanics and Engineering, 29, 329-349. 
[101] Kalro, V.\& Tezduyar, T.E. (2000). A Parallel 3D Computational Method for FluidStructure Interactions in Parachute Systems. Computer Methods in Applied Mechanics and Engineering, 190, 321-332.

[102] Bazilevs, Y., Hsu, M.C., Kiendl, J., Wüchner, R., \& Bletzinger, K.U. (2011). 3D simulation of wind turbine rotors at full scale. Part II: Fluid-structure interaction modeling with composite blades. International Journal for Numerical Methods in Fluids, 65, 236-253.

[103] Hsu, M.C., Akkerman, I., \& Bazilevs, Y. (2011). High-performance computing of wind turbine aerodynamics using isogeometric analysis. Computers and Fluids, 49, $93-100$.

[104] Bazilevs, Y., Hsu, M.C., \& Scott, M.A. (2012). Isogeometric Fluid-Structure Interaction Analysis with Emphasis on NonMatching Discretizations, and with Application to Wind Turbines. Computer Methods in Applied Mechanics and Engineering, 249-252, 28-41.

[105] Hsu, M.C., Akkerman, I., \& Bazilevs, Y. (2014). Finite element simulation of wind turbine aerodynamics: Validation study using NREL Phase VI experiment. Wind Energy, 17, 461-481.

[106] Korobenko, A., Hsu, M.C., Akkerman, I., Tippmann, J., \& Bazilevs, Y. (2013). Structural mechanics modeling and FSI simulation of wind turbines. Mathematical Models and Methods in Applied Sciences, 23, 249-272.

[107] Bazilevs, Y., Korobenko, A., Deng, X., \& Yan, J. (2015). Novel structural modeling and mesh moving techniques for advanced FSI simulation of wind turbines. International Journal for Numerical Methods in Engineering, 102, 766-783.

[108] Korobenko, A., Yan, J., Gohari, S.M.I., Sarkar, S., \& Bazilevs, Y. (2017). FSI simulation of two back-to-back wind turbines in atmospheric boundary layer flow. Computers 65 Fluids, 158, 167-175.
[109] Korobenko, A., Hsu, M.C., Akkerman, I., \& Bazilevs, Y. (2013). Aerodynamic simulation of vertical-axis wind turbines. Journal of Applied Mechanics, 81, 021011.

[110] Bazilevs, Y., Korobenko, A., Deng, X., Yan, J., Kinzel, M., \& Dabiri, J.O. (2014). FSI modeling of vertical-axis wind turbines. Journal of Applied Mechanics, 81, 081006 .

[111] Yan, J., Korobenko, A., Deng, X., \& Bazilevs, Y. (2016). Computational freesurface fluid-structure interaction with application to floating offshore wind turbines. Computers and Fluids, 141, 155174.

[112] Bazilevs, Y., Korobenko, A., Yan, J., Pal, A., Gohari, S.M.I., \& Sarkar, S. (2015). ALE-VMS Formulation for Stratified Turbulent Incompressible Flows with Applications. Mathematical Models and Methods in Applied Sciences, 25, 2349-2375.

[113] Bazilevs, Y., Korobenko, A., Deng, X., \& Yan, J. (2016). FSI modeling for fatiguedamage prediction in full-scale windturbine blades. Journal of Applied $\mathrm{Me}$ chanics, 83(6), 061010.

[114] Bazilevs, Y., Calo, V.M., Zhang, Y., \& Hughes, T.J.R. (2006). Isogeometric fluidstructure interaction analysis with applications to arterial blood flow. Computational Mechanics, 38, 310-322.

[115] Bazilevs, Y., Gohean, J.R., Hughes, T.J.R., Moser, R.D., \& Zhang, Y. (2009). Patient-specific isogeometric fluidstructure interaction analysis of thoracic aortic blood flow due to implantation of the Jarvik 2000 left ventricular assist device. Computer Methods in Applied $\mathrm{Me}$ chanics and Engineering, 198, 3534-3550.

[116] Bazilevs, Y., Hsu, M.C., Benson, D., Sankaran, S., \& Marsden, A. (2009). Computational Fluid-Structure Interaction: Methods and Application to a Total Cavopulmonary Connection. Computational Mechanics, 45, 77-89. 
[117] Bazilevs, Y., Hsu, M.C., Zhang, Y., Wang, W., Liang, X., Kvamsdal, T., Brekken, R., \& Isaksen, J. (2010). A Fully-Coupled Fluid-Structure Interaction Simulation of Cerebral Aneurysms. Computational Mechanics, 46, 3-16.

[118] Bazilevs, Y., Hsu, M.C., Zhang, Y., Wang, W., Kvamsdal, T., Hentschel, S., \& Isaksen, J. (2010). Computational FluidStructure Interaction: Methods and Application to Cerebral Aneurysms. Biomechanics and Modeling in Mechanobiology, 9, 481-498.

[119] Hsu, M.C. \& Bazilevs, Y. (2011). Blood vessel tissue prestress modeling for vascular fluid-structure interaction simulations. Finite Elements in Analysis and Design, 47, 593-599.

[120] Long, C.C., Marsden, A.L., \& Bazilevs, Y. (2013). Fluid-structure interaction simulation of pulsatile ventricular assist devices. Computational Mechanics, 52, 971981.

[121] Long, C.C., Esmaily-Moghadam, M., Marsden, A.L., \& Bazilevs, Y. (2014). Computation of residence time in the simulation of pulsatile ventricular assist devices. Computational Mechanics, 54, 911919.

[122] Long, C.C., Marsden, A.L., \& Bazilevs, Y. (2014). Shape optimization of pulsatile ventricular assist devices using FSI to minimize thrombotic risk. Computational $\mathrm{Me}$ chanics, 54, 921-932.

[123] Hsu, M.C., Kamensky, D., Xu, F., Kiendl, J., Wang, C., Wu, M.C.H., Mineroff, J., Reali, A., Bazilevs, Y., \& Sacks, M.S. (2015). Dynamic and fluid-structure interaction simulations of bioprosthetic heart valves using parametric design with $\mathrm{T}$ splines and Fung-type material models. Computational Mechanics, 55, 1211-1225.

[124] Akkerman, I., Bazilevs, Y., Benson, D.J., Farthing, M.W., \& Kees, C.E. (2012). Free-Surface Flow and Fluid-Object Interaction Modeling with Emphasis on Ship
Hydrodynamics. Journal of Applied Mechanics, 79, 010905.

[125] Akkerman, I., Dunaway, J., Kvandal, J., Spinks, J., \& Bazilevs, Y. (2012). Toward free-surface modeling of planing vessels: simulation of the Fridsma hull using ALEVMS. Computational Mechanics, 50, 719727.

[126] Wang, C., Wu, M.C.H., Xu, F., Hsu, M.C., \& Bazilevs, Y. (2017). Modeling of a hydraulic arresting gear using fluid-structure interaction and isogeometric analysis. Computers and Fluids, 142, $3-14$.

[127] Wu, M.C.H., Kamensky, D., Wang, C., Herrema, A.J., Xu, F., Pigazzini, M.S., Verma, A., Marsden, A.L., Bazilevs, Y., \& Hsu, M.C. (2017). Optimizing fluidstructure interaction systems with immersogeometric analysis and surrogate modeling: Application to a hydraulic arresting gear. Computer Methods in Applied $\mathrm{Me}$ chanics and Engineering, 316, 668-693.

[128] Yan, J., Deng, X., Korobenko, A., \& Bazilevs, Y. (2017). Free-surface flow modeling and simulation of horizontal-axis tidal-stream turbines. Computers and Fluids, 158, 157-166.

[129] Castorrini, A., Corsini, A., Rispoli, F., Takizawa, K., \& Tezduyar, T.E. (February 2019). A stabilized ALE method for computational fluid-structure interaction analysis of passive morphing in turbomachinery, Mathematical Models and Methods in Applied Sciences, published online, DOI: $10.1142 /$ S0218202519410057.

[130] Augier, B., Yan, J., Korobenko, A., Czarnowski, J., Ketterman, G., \& Bazilevs, Y. (2015). Experimental and numerical FSI study of compliant hydrofoils. Computational Mechanics, 55, 1079-1090.

[131] Yan, J., Augier, B., Korobenko, A., Czarnowski, J., Ketterman, G., \& Bazilevs, Y. (2016). FSI modeling of a propulsion system based on compliant hydrofoils in a tandem configuration. Computers and Fluids, 141, 201-211. 
[132] Helgedagsrud, T.A., Bazilevs, Y., Mathisen, K.M., \& Oiseth, O.A. Computational and experimental investigation of free vibration and flutter of bridge decks. Computational Mechanics, published online.

[133] Helgedagsrud, T.A., Bazilevs, Y., Korobenko, A., Mathisen, K.M., \& Oiseth, O.A. Using ALE-VMS to compute aerodynamic derivatives of bridge sections. Computers and Fluids, published online.

[134] Helgedagsrud, T.A., Akkerman, I., Bazilevs, Y., Mathisen, K.M., \& Oiseth, O.A. Isogeometric modeling and experimental investigation of moving-domain bridge aerodynamics. ASCE Journal of Engineering Mechanics, accepted for publication.

[135] Yan, J., Korobenko, A., Tejada-Martinez, A.E., Golshan, R., \& Bazilevs, Y. (2017). A new variational multiscale formulation for stratified incompressible turbulent flows. Computers \& Fluids, 158, 150-156.

[136] van Opstal, T.M., Yan, J., Coley, C., Evans, J.A., Kvamsdal, T., \& Bazilevs, Y. (2017). Isogeometric divergenceconforming variational multiscale formulation of incompressible turbulent flows. Computer Methods in Applied Mechanics and Engineering, 316, 859-879.

[137] Xu, F., Moutsanidis, G., Kamensky, D., Hsu, M.C., Murugan, M., Ghoshal, A., \& Bazilevs, Y. (2017). Compressible flows on moving domains: Stabilized methods, weakly enforced essential boundary conditions, sliding interfaces, and application to gas-turbine modeling. Computers \& $\mathrm{Flu}$ ids, 158, 201-220.

[138] Hsu, M.C. \& Kamensky, D. (2018). Immersogeometric Analysis of Bioprosthetic Heart Valves, Using the Dynamic Augmented Lagrangian Method. In Tezduyar, T.E. (editor), Frontiers in Computational Fluid-Structure Interaction and Flow Simulation, Cham: Springer International Publishing, 167-212.
[139] Tezduyar, T.E., Takizawa, K., Moorman, C., Wright, S., \& Christopher, J. (2010). Space-Time Finite Element Computation of Complex Fluid-Structure Interactions. International Journal for $\mathrm{Nu}$ merical Methods in Fluids, 64, 1201-1218.

[140] Kiendl, J., Hsu, M.C., Wu, M.C.H., \& Reali, A. (2015). Isogeometric KirchhoffLove shell formulations for general hyperelastic materials. Computer Methods in Applied Mechanics and Engineering, 291, 280-303.

[141] Wu, M.C.H., Zakerzadeh, R., Kamensky, D., Kiendl, J., Sacks, M.S., \& Hsu, M.C. (2018). An anisotropic constitutive model for immersogeometric fluid-structure interaction analysis of bioprosthetic heart valves. Journal of Biomechanics, 74, 2331.

[142] Kamensky, D., Hsu, M.C., Yu, Y., Evans, J.A., Sacks, M.S., \& Hughes, T.J.R. (2017). Immersogeometric cardiovascular fluid-structure interaction analysis with divergence-conforming B-splines. Computer Methods in Applied Mechanics and Engineering, 314, 408-472.

[143] Kamensky, D., Evans, J.A., \& Hsu, M.C. (2015). Stability and Conservation Properties of Collocated Constraints in Immersogeometric Fluid-Thin Structure Interaction Analysis. Communications in Computational Physics, 18(04), 1147-1180.

[144] Kamensky, D., Evans, J.A., Hsu, M.C., \& Bazilevs, Y. (2017). Projection-based stabilization of interface Lagrange multipliers in immersogeometric fluid-thin structure interaction analysis, with application to heart valve modeling. Computers and Mathematics with Applications, 74, 20682088.

[145] Yu, Y., Kamensky, D., Hsu, M.C., Lu, X.Y., Bazilevs, Y., \& Hughes, T.J.R. (2018). Error estimates for projectionbased dynamic augmented Lagrangian boundary condition enforcement, with application to fluid-structure interaction. Mathematical Models and Methods in Applied Science, 28, 2457-2509. 
[146] Xu, F., Morganti, S., Zakerzadeh, R., Kamensky, D., Auricchio, F., Reali, A., Hughes, T.J.R., Sacks, M.S., \& Hsu, M.C. (2018). A framework for designing patientspecific bioprosthetic heart valves using immersogeometric fluid-structure interaction analysis. International Journal for Numerical Methods in Biomedical Engineering, 34(4), e2938.

[147] Tezduyar, T.E., Aliabadi, S.K., Behr, M., \& Mittal, S. (1994). Massively Parallel Finite Element Simulation of Compressible and Incompressible Flows. Computer Methods in Applied Mechanics and Engineering, 119, 157-177.

[148] Tezduyar, T.E. \& Ganjoo, D.K. (1986). Petrov-Galerkin Formulations with Weighting Functions Dependent upon Spatial and Temporal Discretization: Applications to Transient ConvectionDiffusion Problems. Computer Methods in Applied Mechanics and Engineering, 59, 49-71.

[149] Le Beau, G.J., Ray, S.E., Aliabadi, S.K., \& Tezduyar, T.E. (1993). SUPG Finite Element Computation of Compressible Flows with the Entropy and Conservation Variables Formulations. Computer Methods in Applied Mechanics and Engineering, 104, 397-422.

[150] Tezduyar, T.E. \& Senga, M. (2006). Stabilization and Shock-Capturing Parameters in SUPG Formulation of Compressible Flows. Computer Methods in Applied Mechanics and Engineering, 195, 1621-1632.

[151] Corsini, A., Menichini, C., Rispoli, F., Santoriello, A., \& Tezduyar, T.E. (2009). A Multiscale Finite Element Formulation with Discontinuity Capturing for Turbulence Models with Dominant Reactionlike Terms. Journal of Applied Mechanics, 76, 021211.

[152] Rispoli, F., Saavedra, R., Menichini, F., \& Tezduyar, T.E. (2009). Computation of Inviscid Supersonic Flows around Cylinders and Spheres with the V-SGS Stabilization and YZ $\beta$ Shock-Capturing. Journal of Applied Mechanics, 76, 021209.
[153] Corsini, A., Iossa, C., Rispoli, F., \& Tezduyar, T.E. (2010). A DRD Finite Element Formulation for Computing Turbulent Reacting Flows in Gas Turbine Combustors. Computational Mechanics, 46 , 159-167.

[154] Hsu, M.C., Bazilevs, Y., Calo, V.M., Tezduyar, T.E., \& Hughes, T.J.R. (2010). Improving Stability of Stabilized and Multiscale Formulations in Flow Simulations at Small Time Steps. Computer Methods in Applied Mechanics and Engineering, 199, 828-840.

[155] Corsini, A., Rispoli, F., \& Tezduyar, T.E. (2011). Stabilized Finite Element Computation of NOx Emission in Aeroengine Combustors. International Journal for Numerical Methods in Fluids, 65, 254270.

[156] Corsini, A., Rispoli, F., \& Tezduyar, T.E. (2012). Computer modeling of waveenergy air turbines with the SUPG/PSPG formulation and discontinuity-capturing technique. Journal of Applied Mechanics, 79, 010910.

[157] Corsini, A., Rispoli, F., Sheard, A.G., \& Tezduyar, T.E. (2012). Computational Analysis of Noise Reduction Devices in Axial Fans with Stabilized Finite Element Formulations. Computational Mechanics, 50, 695-705.

[158] Kler, P.A., Dalcin, L.D., Paz, R.R., \& Tezduyar, T.E. (2013). SUPG and discontinuity-capturing methods for coupled fluid mechanics and electrochemical transport problems. Computational $\mathrm{Me}$ chanics, 51, 171-185.

[159] Corsini, A., Rispoli, F., Sheard, A.G., Takizawa, K., Tezduyar, T.E., \& Venturini, P. (2014). A variational multiscale method for particle-cloud tracking in turbomachinery flows. Computational $\mathrm{Me}$ chanics, 54, 1191-1202.

[160] Rispoli, F., Delibra, G., Venturini, P., Corsini, A., Saavedra, R., \& Tezduyar, T.E. (2015). Particle tracking and 
particle-shock interaction in compressibleflow computations with the V-SGS stabilization and YZ $\beta$ shock-capturing. Computational Mechanics, 55, 1201-1209.

[161] Cardillo, L., Corsini, A., Delibra, G., Rispoli, F., \& Tezduyar, T.E. (2016). Flow analysis of a wave-energy air turbine with the SUPG/PSPG stabilization and Discontinuity-Capturing Directional Dissipation. Computers \& Fluids, 141, 184190.

[162] Castorrini, A., Corsini, A., Rispoli, F., Venturini, P., Takizawa, K., \& Tezduyar, T.E. (2016). Computational analysis of wind-turbine blade rain erosion. Computers Ef Fluids, 141, 175-183.

[163] Takizawa, K., Tezduyar, T.E., \& Otoguro, Y. (2018). Stabilization and discontinuitycapturing parameters for space-time flow computations with finite element and isogeometric discretizations. Computational Mechanics, 62, 1169-1186.

[164] Castorrini, A., Corsini, A., Rispoli, F., Venturini, P., Takizawa, K., \& Tezduyar, T.E. (March 2019). Computational analysis of performance deterioration of a wind turbine blade strip subjected to environmental erosion, Computational Mechanics, published online, DOI: 10.1007/s00466019-01697-0.

[165] Takizawa, K. \& Tezduyar, T.E. (2014). Space-time computation techniques with continuous representation in time (ST-C). Computational Mechanics, 53, 91-99.

[166] Takizawa, K., Tezduyar, T.E., \& Sasaki, T. (2017). Aorta modeling with the element-based zero-stress state and isogeometric discretization. Computational $\mathrm{Me}$ chanics, 59, 265-280.

[167] Takizawa, K., Tezduyar, T.E., \& Sasaki, T. (2019). Isogeometric hyperelastic shell analysis with out-of-plane deformation mapping. Computational Mechanics, 63, 681-700.

[168] Tezduyar, T.E., Cragin, T., Sathe, S., \& Nanna, B. (2007). FSI Computations in Arterial Fluid Mechanics with Estimated Zero-Pressure Arterial Geometry. In Onate, E., Garcia, J., Bergan, P., \& Kvamsdal, T. (editors), Marine 2007, Barcelona, Spain: CIMNE.

[169] Murray, C.D. (1926). The Physiological Principle of Minimum Work: I. The Vascular System and the Cost of Blood Volume. Proceedings of the National Academy of Sciences of the United States of America, 12, 207-214.

[170] Scott, M.A., Simpson, R.N., Evans, J.A., Lipton, S., Bordas, S.P.A., Hughes, T.J.R., \& Sederberg, T.W. (2013). Isogeometric boundary element analysis using unstructured T-splines. Computer Methods in Applied Mechanics and Engineering, 254, 197-221.

[171] Tezduyar, T., Aliabadi, S., Behr, M., Johnson, A., \& Mittal, S. (1993). Parallel Finite-Element Computation of 3D Flows. Computer, 26(10), 27-36.

[172] Johnson, A.A. \& Tezduyar, T.E. (1994). Mesh Update Strategies in Parallel Finite Element Computations of Flow Problems with Moving Boundaries and Interfaces. Computer Methods in Applied Mechanics and Engineering, 119, 73-94.

[173] Hsu, M.C., Wang, C., Herrema, A.J., Schillinger, D., Ghoshal, A., \& Bazilevs, Y. (2015). An interactive geometry modeling and parametric design platform for isogeometric analysis. Computers and Mathematics with Applications, 70, 1481-1500. 


\section{About Authors}

Kenji TAKIZAWA received his $\mathrm{PhD}$ from Tokyo Institute of Technology in 2005, and he is currently a Professor in Department of Modern Mechanical Engineering at Waseda University. $\mathrm{He}$ has been conducting computational fluid mechanics research since 2000, teaching classes on that subject since 2010, and has been conducting computational FSI research since 2003. He has published over 90 Web-of-Scienceindexed journal articles on computational fluid and structural mechanics and FSI. He is a Web of Science Highly Cited Researcher. He coFluids and is responsibleauthored a textbook titled Computational Fluid-Structure Interaction: Methods and Applications, published by Wiley, with the Japanese translation published by Morikita Publishing Company. He served an Associate Editor of ASME Journal of Applied Mechanics and was responsible for the manuscripts on computational fluid mechanics and FSI. More information on Takizawa can be found at http://www.jp.tafsm.org/.

Yuri BAZILEVS received his $\mathrm{PhD}$ from University of Texas at Austin in 2006, and he is currently the E. Paul Sorensen Professor of Engineering at Brown University. He has been conducting computational fluid mechanics research since 2000, teaching classes on that subject since 2008, and has been conducting computational FSI research since 2005. He has published over 140 Web-of-Science-indexed journal articles on computational fluid and structural mechanics and FSI. He is a Web of Science Highly Cited Researcher. He coauthored a book on isogeometric analysis, a technique widely used in computational mechanics, and FSI. He coauthored a textbook titled Computational Fluid-Structure Interaction: Methods and Applications, published by Wiley, with the Japanese translation published by Morikita Publishing Company. $\mathrm{He}$ is an Associate Editor of Elsevier journal Computers and Fluids and is responsible for the manuscripts on computational fluid mechanics and FSI. More information on Bazilevs can be found at https://vivo.brown.edu/display/ybazilev.
Tayfun E. TEZDUYAR received his $\mathrm{PhD}$ from Caltech in 1982, and he is currently the James F. Barbour Professor of Mechanical Engineering at Rice University. He has been conducting computational fluid mechanics research since 1979, teaching classes on that subject since 1987, and has been conducting computational FSI research since 1991. He has published over 240 Web-of-Science-indexed journal articles on computational fluid and structural mechanics and FSI. He is a Web of Science Highly Cited Researcher. He coauthored a textbook titled Computational Fluid-Structure Interaction: Methods and Applications, published by Wiley, with the Japanese translation published by Morikita Publishing Company. $\mathrm{He}$ is an Editor of Springer journal Computational Mechanics and is responsible for the manuscripts on computational fluid mechanics and FSI. More information on Tezduyar can be found at http://www.tafsm.org/tezduyar/.

Ming-Chen HSU received his $\mathrm{PhD}$ from University of California, San Diego in 2012, and he is currently an Associate Professor in the Department of Mechanical Engineering at Iowa State University. He has been conducting computational fluid mechanics research since 2006, teaching classes on that subject since 2013, and has been conducting computational FSI research since 2008. He has published over 60 Web-of-Science-indexed journal articles on computational fluid and structural mechanics and FSI. He is a Web of Science Highly Cited Researcher. More information on Hsu can be found at https://web.me.iastate.edu/jmchsu/. 